\title{
Socioeconomic inequalities in occupational, leisure-time, and transport related physical activity among European adults: A systematic review
}

Marielle A Beenackers ${ }^{1 *}$, Carlijn BM Kamphuis ${ }^{1}$, Katrina Giskes ${ }^{1,2,3}$, Johannes Brug ${ }^{4}$, Anton E Kunst ${ }^{1,5}$, Alex Burdorf ${ }^{1}$ and Frank J van Lenthe ${ }^{1}$

\begin{abstract}
Background: This study systematically reviewed the evidence pertaining to socioeconomic inequalities in different domains of physical activity (PA) by European region.

Methods: Studies conducted between January 2000 and December 2010 were identified by a systematic search in Pubmed, Embase, Web of Science, Psychinfo, Sportdiscus, Sociological Abstracts, and Social Service Abstracts.

English-language peer-reviewed studies undertaken in the general population of adults (18-65 years) were classified by domain of PA (total, leisure-time including sport, occupational, active transport), indicator of socioeconomic position (education, income, occupation), and European region. Distributions of reported positive, negative, and null associations were evaluated.

Results: A total of 131 studies met the inclusion criteria. Most studies were conducted in Scandinavia $(n=47)$. Leisure-time PA was the most frequently studied PA outcome $(n=112)$. Considerable differences in the direction of inequalities were seen for the different domains of PA. Most studies reported that those with high socioeconomic position were more physically active during leisure-time compared to those with low socioeconomic position (68\% positive associations for total leisure-time PA, 76\% for vigorous leisure-time PA). Occupational PA was more prevalent among the lower socioeconomic groups (63\% negative associations). Socioeconomic differences in total PA and active transport PA did not show a consistent pattern (40\% and 38\% positive associations respectively). Some inequalities differed by European region or socioeconomic indicator, however these differences were not very pronounced.
\end{abstract}

Conclusions: The direction of socioeconomic inequalities in PA in Europe differed considerably by domain of PA. The contradictory results for total PA may partly be explained by contrasting socioeconomic patterns for leisure-time PA and occupational PA.

Keywords: Socioeconomic, Inequalities, Physical activity, Systematic review, Europe

\footnotetext{
* Correspondence: m.beenackers@erasmusmc.nl

'Department of Public Health, Erasmus MC, Rotterdam, Netherlands

Full list of author information is available at the end of the article
} 


\section{Introduction}

Socioeconomic inequalities in morbidity and mortality are well-documented [1,2]. Differences in health behaviours play an important role in these inequalities [3]. Next to the higher prevalence of smoking in lower socio-economic groups [4,5], evidence suggests that the higher obesity rates are of major importance to health inequalities [6-9].

Obesity levels in Europe are rising rapidly; the prevalence of obesity has tripled since the 1980s [10]. This high prevalence of obesity is estimated to account for 1 million deaths and 12 million life years of ill health in Europe each year [10]. European regions are thought to be in a different stage of the obesity epidemic; when the level of economic development increases, the proportion of positive associations between socioeconomic position (SEP) and overweight and obesity decreases and the proportion of negative association increases [6,7]. Because overweight and obesity are the result of an excessive energy intake or limited energy expenditure, differences in dietary intake or physical activity (PA) are expected to contribute to the socioeconomic inequalities in overweight and obesity. A recent review of socioeconomic inequalities in nutrition in Europe [11] reported that consistent socioeconomic inequalities in diet were seen for fruit and vegetable consumption and, to a lesser degree, for fibre consumption but not in amounts of energy intake. PA is a health behaviour of major importance as it is strongly associated with obesity and a number of diseases such as metabolic disease and certain cancers $[12,13]$. However, no systematic review of the evidence of socio-economic differences in PA in Europe has been published to date.

PA is often categorized as low intensity PA $(<3$ Metabolic Equivalent (MET)) versus moderate (3-6 METs) to vigorous PA ( $>6$ METs) [14]. The latter two categories are regarded as especially important for health. Furthermore, leisure-time, work-related, and transport-related PA are often distinguished from each other. Empirical evidence suggests that socioeconomic patterns may differ for different domains of PA $[15,16]$. Patterns may also differ by gender, as exemplified by the finding that inequalities in overweight and obesity are larger in women [7], and by European region, as illustrated by the North to South gradient in obesity inequalities [6,7]. Finally, traditional indicators of SEP, such as income, occupation, and education, may reflect different aspects of one's position in the social stratification $[17,18]$, and may therefore be more strongly or weakly related to specific outcomes.

The purpose of this review is to describe socioeconomic inequalities in different domains of physical activity, across different SEP indicators, in men and women, and across different regions in Europe.

\section{Method}

\section{Search strategy}

\section{Databases and search terms}

Major databases (PubMed, EMbase, Web of Science, PsychINFO, SportDiscus, and Sociological Abstracts and Social Services Abstracts) were searched to locate relevant studies published between the first of January 2000 and the $31^{\text {st }}$ of December 2010. Broad search terms, including synonyms, were used to ensure that all potentially relevant articles were included in the search results. When possible, database specific search terms were used to optimize the results. The search strategy and syntax for each database are available from the authors (MAB) upon request.

\section{Inclusion and exclusion criteria}

Publications were included if they were published in English-written peer-reviewed journals. Studies had to be conducted among the general population, which therefore excluded studies utilizing patient groups. Given the interest in occupational physical activity, study participants had to be of working age (18-65 years of age). Studies quantitatively assessed the association between at least one SEP indicator and one domain of physical activity (measured, either in terms of frequency (e.g. times/week), duration (e.g. hours or minutes), and/ or intensity (e.g. vigorous)). Indicators of SEP included education, social class (based on occupation), income (either individual or household level), household wealth (e.g. car ownership, housing tenure) or area-based indicators (e.g. area deprivation). Outcomes included were total physical activity, leisure-time physical activity including but not limited to sports and exercise (both organized and unorganized), active transport (walking, cycling), and occupational physical activity. Manuscripts that elicited concerns about the study quality were excluded. These quality concerns were inconsistencies between the results in the text and the results in the tables, missing information on how the outcome or SEP indicator was measured, or missing information on the basic description of the sample, such as sample size.

\section{Data extraction and summarization Title scanning and selection}

As a first step in identifying relevant studies, titles and abstracts were read by the lead author (MAB). Second, the full text was read if studies met the inclusion criteria and when it was clear from the title and/or abstract that the association between SEP and PA was studied. A second researcher (CBMK) executed an independent parallel selection process with a random subsample of 200 titles and abstracts which resulted in a similar selection. 


\section{Data synthesis}

The following information was extracted into data extraction tables from each included study: country, year (or years) the data were collected, sample size and sample characteristics (in case a subpopulation was studied), age range, percentage males, percentage response, SEP indicator and PA outcomes (Table 1).

\section{Classification of the outcome measures}

The following guidelines were used to classify the studies into the different domains of PA:

- A PA outcome was categorized as 'total physical activity' (TPA) if it concerned a general PA question (not defined whether they mean occupational PA or leisure-time PA) or if the measure included leisuretime PA as well as occupational PA. Total physical activity was often described as 'usual' or 'daily' physical activity.

- A PA outcome was categorized as 'occupational physical activity' (OPA) if it was specifically identified as occupational PA in the methods with words such as 'occupational' or 'during work'.

- A PA outcome was categorized as 'total leisure-time physical activity' (TLTPA) if it was specifically identified as leisure-time PA in the methods with words such as 'in free time' or 'during leisure time'. Exception: leisure-time physical activity that can be defined as vigorous physical activity (see classification criteria below).

- A PA outcome was categorized as 'vigorous leisuretime physical activity' (VLTPA) if the methods specifically reported that it is about high intensity physical activity, vigorous physical activity, conditioning physical activity, or sports participation. Only vigorous physical activity at leisure time was considered for this category.

- A PA outcome was categorized as 'active transport' (AT) if the outcome measure was defined as walking or cycling to work, school or other destinations such as shops or friends.

For some studies, PA outcomes could not be clearly classified in either of these groups (e.g. heavy manual leisure (like chopping wood) or walking or cycling of which the purpose (leisure or transport) was not clear). Therefore, these outcomes were excluded from the current review.

\section{Classification of the socioeconomic position indicators}

The following guidelines were used to classify the SEP indicators in this study.

- Income refers to (net or gross) individual income or household income. When area-level income was used as an indicator, it was classified as 'other' and specified further in the footnotes of the tables.

- Education refers to the highest attained level of education (e.g. university education) or as the total years of education.

- Social class refers to occupation-based social class, such as blue collar or white collar workers, or the British Registrar General classification [19].

- Other SEP indicators that were included were neighbourhood SEP, such as mean/median income of a neighbourhood, material circumstances, such as home ownership, or other individual SEP measures, such as an individual composite SEP score that was constructed from several SEP indicators.

Parental SEP, childhood SEP, or the SEP of the spouse were excluded as a SEP indicator in this review.

\section{Classification of European regions}

The results were grouped by European region, based on geographical location and type of welfare regime $[20,21]$. The regions that were distinguished are:

- Anglo-Saxon region, including Great-Britain and Ireland

- Western European region, including Belgium, France, Germany, Luxembourg, Netherlands, and Switzerland

- Scandinavian region, including Denmark, Finland, Norway, and Sweden

- Southern European region, including Greece, Italy, Portugal, and Spain

- Eastern European region, including Albania, Croatia (Hrvatska), Czech Republic, Estonia, Hungary, Latvia, Lithuania, Poland, and Slovenia

As many studies included more than one PA domain and/or more than one SEP indicator, the results were analysed on the level of the separate associations rather than the level of complete studies. This is in concordance with methods form McLaren [6] and Ball and Crawford [22]. The advantage is that we could distinguish between the domains of PA behaviour and the SEP indicators. Disadvantages of this method are that all associations are weighted equally and that studies with more associations have more influence than those with only one reported association [6].

Detailed tables in which all the associations reported in the included studies were synthesized are described in the additional tables (Additional file 1, tables A1-A5, one for each domain of PA). A ' + ' indicates a positive and significant association between the SEP indicator and the PA outcome of interest, a '-' indicates a negative and significant association between the SEP indicator and 
Table 1 Characteristics of the 131 included studies ordered by European region

\begin{tabular}{|c|c|c|c|c|c|c|c|c|c|}
\hline $\begin{array}{l}\text { Author, year of } \\
\text { publication }\end{array}$ & Country of study ${ }^{a}$ & Study name & $\begin{array}{l}\text { Year of data- } \\
\text { collection }\end{array}$ & $\begin{array}{l}\text { Sample size }+ \\
\text { characteristic }^{\text {b }}\end{array}$ & Age & $\%$ Male $^{c}$ & Response $^{c}$ & SEP indicator ${ }^{d}$ & PA domain ${ }^{e}$ \\
\hline \multicolumn{10}{|l|}{ EU wide studies } \\
\hline $\begin{array}{l}\text { Martinez-Gonzales } \\
\text { et al., } 2001 \text { [54] }\end{array}$ & EU - 15 countries & Pan-European Union survey & 1997 & $n=15239$ & $15+$ & $47 \%$ & NP & Education & TLTPA \\
\hline Ståhl et al., 2001 [60] & $\begin{array}{l}\text { BEL, FIN, DEU, NLD, } \\
\text { ESP, SWI }\end{array}$ & MAREPS project & 1997-98 & $n=3343$ & $18+$ & $43 \%$ & $54 \%$ & Education & TPA \\
\hline $\begin{array}{l}\text { Van Tuyckom \& } \\
\text { Scheerder, } 2008 \text { [61] }\end{array}$ & EU - 27 countries & Eurobarometer 64.3 & 2005 & $n=26688$ & $15+$ & NP & NP & Education Social class & TLTPA \\
\hline $\begin{array}{l}\text { Van Tuyckom \& } \\
\text { Scheerder, 2010a } \\
\text { [62] }\end{array}$ & EU - 27 countries & Eurobarometer 64.3 & 2005 & $n=26362$ & $15+$ & NP & NP & Social class & TLTPA \\
\hline $\begin{array}{l}\text { Van Tuyckom \& } \\
\text { Scheerder, 2010b } \\
\text { [55] }\end{array}$ & EU - 27 countries & Eurobarometer 64.3 & 2005 & $n=26688$ & $15+$ & NP & NP & Education & OPA TLTPA AT \\
\hline Varo et al., 2003 [56] & EU - 15 countries & Pan-European Union survey & 1997 & $n=15239$ & $15+$ & $47 \%$ & NP & Education & TLTPA \\
\hline \multicolumn{10}{|l|}{$\begin{array}{l}\text { Western European } \\
\text { region }\end{array}$} \\
\hline $\begin{array}{l}\text { Addor et al., } 2003 \\
\text { [63] }\end{array}$ & SWI & $\begin{array}{l}\text { Health examination survey of } \\
\text { adults (MONICA project) }\end{array}$ & 1992-93 & $n=1550$ & $25-64$ & $49 \%$ & $53 \%$ & Education & VLTPA \\
\hline $\begin{array}{l}\text { Bertrais et al., } 2004 \\
\text { [64] }\end{array}$ & FRA & SUVIMAX study & 1998 & $n=7404$ & $45-68$ & $46 \%$ & NP & Education & TLTPA \\
\hline $\begin{array}{l}\text { Chaix \& Chauvin, } \\
2003[65]\end{array}$ & FRA & $\begin{array}{l}2000 \text { French Health } \\
\text { Monitoring Survey }\end{array}$ & 2000 & $n=12948$ & $16+$ & $49 \%$ & $66 \%$ & Education Income & TLTPA \\
\hline \multirow{2}{*}{$\begin{array}{l}\text { Dragano et al., } \\
2007 \text { [66] }\end{array}$} & \multirow[t]{2}{*}{ DEU, CZE } & \multirow{2}{*}{$\begin{array}{l}\text { DEU: Heinz Nixdorf Recall } \\
\text { (HNR) Study } \\
\text { CZE: Health, Alcohol \& } \\
\text { Psychosocial Factors in } \\
\text { Eastern Europe (HAPIEE) }\end{array}$} & DEU: 2000-03 & $\mathrm{n}$ DEU $=4032$ & \multirow[t]{2}{*}{$45-69$} & DEU: $49 \%$ & DEU: $56 \%$ & \multirow[t]{2}{*}{ Education Neighb. SEP } & \multirow[t]{2}{*}{ TLTPA } \\
\hline & & & CZE: 2002-05 & n CZE $=7522$ & & CZE: 45\% & CZE: $55 \%$ & & \\
\hline \multirow{3}{*}{$\begin{array}{l}\text { Drieskens et al., } \\
2010[67]\end{array}$} & \multirow[t]{3}{*}{ BEL } & \multirow{3}{*}{$\begin{array}{l}\text { Belgian Health Interview } \\
\text { Survey (HIS) }\end{array}$} & 1997 & n $1997=7431$ & \multirow[t]{3}{*}{$15+$} & \multirow[t]{3}{*}{ NP } & \multirow[t]{3}{*}{$60 \%$} & \multirow[t]{3}{*}{ Education } & \multirow[t]{3}{*}{ TLTPA } \\
\hline & & & 2001 & n $2001=8142$ & & & & & \\
\hline & & & 2004 & n $2004=7459$ & & & & & \\
\hline $\begin{array}{l}\text { Galobardes et al., } \\
2003 \text { [68] }\end{array}$ & SWI & Bus Santé 1993-2000 & 1993-2000 & $n=8194$ & $35-74$ & $51 \%$ & $57-65 \%$ & Education Social class & VLTPA \\
\hline $\begin{array}{l}\text { Kamphuis et al., } \\
2008 \text { [52] }\end{array}$ & NLD & Dutch GLOBE study 2004 & 2004 & $n=3839$ & $25-75$ & $48 \%$ & $64 \%$ & Income Education & VLTPA \\
\hline $\begin{array}{l}\text { Kamphuis et al., } \\
2009 \text { [51] }\end{array}$ & NLD & Dutch GLOBE study 2004 & 2004 & $n=1994$ & $55-75$ & $48 \%$ & $62 \%$ & Income Education & TLTPA \\
\hline $\begin{array}{l}\text { van Lenthe et al., } \\
2005 \text { [47] }\end{array}$ & NLD & Dutch GLOBE study 1991 & 1991 & $n=8767$ & $20-69$ & NP & $70 \%$ & Neighb. SEP & $\begin{array}{l}\text { TLTPA } \\
\text { VLTPA AT }\end{array}$ \\
\hline
\end{tabular}


Table 1 Characteristics of the 131 included studies ordered by European region (Continued)

\begin{tabular}{|c|c|c|c|c|c|c|c|c|c|}
\hline \multirow[t]{2}{*}{$\begin{array}{l}\text { Meyer et al., } 2005 \\
\text { [69] }\end{array}$} & \multirow[t]{2}{*}{ SWI } & \multirow[t]{2}{*}{ Swiss Health Survey 2002} & \multirow[t]{2}{*}{2002} & $\mathrm{n}=8405$ & \multirow[t]{2}{*}{$50+$} & \multirow[t]{2}{*}{$45 \%$} & \multirow[t]{2}{*}{ NP } & \multirow[t]{2}{*}{ Income Education } & \multirow[t]{2}{*}{ VLTPA } \\
\hline & & & & $\begin{array}{l}\text { community } \\
\text { residents }\end{array}$ & & & & & \\
\hline $\begin{array}{l}\text { Nocon et al., } 2008 \\
\text { [70] }\end{array}$ & DEU & German National Health Survey & 1998 & $n=7124$ & $18-79$ & $48 \%$ & NP & $\begin{array}{l}\text { Income Education Social } \\
\text { class }\end{array}$ & VLTPA \\
\hline $\begin{array}{l}\text { van Oort et al., } 2004 \\
\text { [71] }\end{array}$ & NLD & Dutch GLOBE study 1991 & 1991 & $n=16980$ & $15-74$ & $49 \%$ & $70 \%$ & Education & TLTPA \\
\hline $\begin{array}{l}\text { Rathmann et al., } \\
2005 \text { [72] }\end{array}$ & DEU & $\begin{array}{l}\text { KORA (=Cooperative Health } \\
\text { Research in the Region of } \\
\text { Augsburg) Survey }\end{array}$ & 2000 & $n=1653$ & $55-74$ & $51 \%$ & $62 \%$ & $\begin{array}{l}\text { Income Education Social } \\
\text { class }\end{array}$ & TLTPA \\
\hline $\begin{array}{l}\text { Ribet et al., } 2001 \\
\text { [73] }\end{array}$ & FRA & $\begin{array}{l}\text { GAZEL study (G)MONICA - } \\
\text { France }(\mathrm{M})\end{array}$ & $\begin{array}{l}\text { G: 1989-92M: } \\
\text { 1994-97 }\end{array}$ & $\begin{array}{l}n G=9486 n \\
M=534 \text { working, } \\
\text { living in couple }\end{array}$ & $40-50$ & $100 \%$ & $\begin{array}{l}\text { G: } 44-87 \% \\
\text { M: } 51-77 \%\end{array}$ & Social class & TLTPA \\
\hline \multirow[t]{5}{*}{$\begin{array}{l}\text { Scheerder et al., } \\
2002[74]\end{array}$} & BEL & Sports participation in Flanders & $\begin{array}{l}19691979 \\
19891999\end{array}$ & n $1969=7479$ & NP & $50 \%$ & $71-89 \%$ & Education Social class & VLTPA \\
\hline & & $\begin{array}{l}\text { - Leuven Growth Study of Flemish } \\
\text { Girls }\end{array}$ & & n $1979=18629$ & & & & & \\
\hline & & $\begin{array}{l}\text { - Study on Movement Activities } \\
\text { in Flanders }\end{array}$ & & n $1989=7957$ & & & & & \\
\hline & & & & n $1999=9143$ & & & & & \\
\hline & & & & $\begin{array}{l}\text { parents of school } \\
\text { children }\end{array}$ & & & & & \\
\hline \multirow[t]{4}{*}{$\begin{array}{l}\text { Scheerder et al., } \\
2005[75]\end{array}$} & BEL & Sports participation in Flanders & $\begin{array}{l}19791989 \\
1999\end{array}$ & n $1979=19396$ & NP & $50 \%$ & $71-89 \%$ & Education Social class & VLTPA \\
\hline & & $\begin{array}{l}\text { - Leuven Growth Study of Flemish } \\
\text { Girls }\end{array}$ & & n $1989=8624$ & & & & & \\
\hline & & $\begin{array}{l}\text { - Study on Movement Activities in } \\
\text { Flanders }\end{array}$ & & n $1999=10356$ & & & & & \\
\hline & & & & $\begin{array}{l}\text { parents of school } \\
\text { children }\end{array}$ & & & & & \\
\hline $\begin{array}{l}\text { Schneider \& Becker, } \\
2005 \text { [76] }\end{array}$ & DEU & German National Health Survey & 1998 & $\mathrm{n}=3323$ employed & $18-69$ & $56 \%$ & $61 \%$ & $\begin{array}{l}\text { Income Education Social } \\
\text { class Individual SEP }\end{array}$ & VLTPA \\
\hline $\begin{array}{l}\text { Van Dyck et al., } \\
2010 \text { [23] }\end{array}$ & BEL & $\begin{array}{l}\text { Belgian Environmental Physical } \\
\text { Activity Study (BEPAS) }\end{array}$ & $2007-08$ & $n=1166$ & $20-65$ & $48 \%$ & $58 \%$ & Education Neighb. SEP & TLTPA AT \\
\hline $\begin{array}{l}\text { Verdaet et al., } \\
2004[77]\end{array}$ & BEL & BELSTRESS study (subsample) & NP & $\begin{array}{l}\mathrm{n}=892 \text { working } \\
\text { men }\end{array}$ & $35-59$ & $100 \%$ & NP & Education & TLTPA \\
\hline $\begin{array}{l}\text { de Vries et al., } \\
2008 \text { [78] }\end{array}$ & NLD & SMILE study & 2002 & $n=9449$ & $12+$ & $42 \%$ & NP & Education & TPA \\
\hline $\begin{array}{l}\text { Wagner et al., } \\
2003 \text { [79] }\end{array}$ & FRA IRE & PRIME Study - France & NP & $\mathrm{n} F \mathrm{FA}=7359$ & $50-59$ & $100 \%$ & NP & $\begin{array}{l}\text { Education Household } \\
\text { wealth }\end{array}$ & $\begin{array}{l}\text { TLTPA VLTPA } \\
\text { AT }\end{array}$ \\
\hline
\end{tabular}


Table 1 Characteristics of the 131 included studies ordered by European region (Continued)

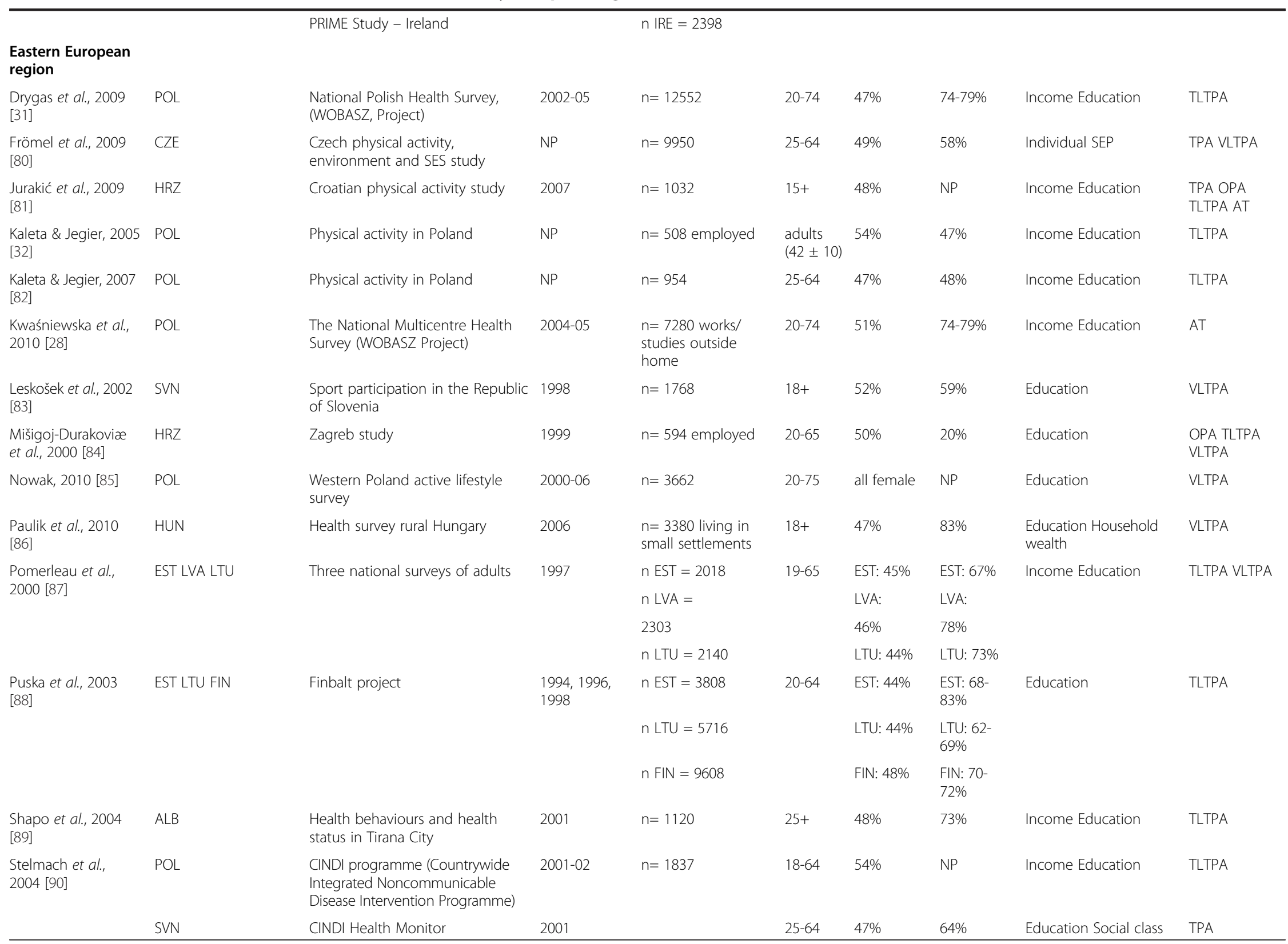


Table 1 Characteristics of the 131 included studies ordered by European region (Continued)

\begin{tabular}{|c|c|c|c|c|c|c|c|c|c|}
\hline $\begin{array}{l}\text { Zaletel-Kragelj et al., } \\
2006 \text { [91] }\end{array}$ & & & & $\begin{array}{l}\mathrm{n}=7718 \text { without } \\
\text { disability }\end{array}$ & & & & & \\
\hline \multicolumn{10}{|l|}{$\begin{array}{l}\text { Southern European } \\
\text { region }\end{array}$} \\
\hline $\begin{array}{l}\text { Artazcoz et al., } 2004 \\
\text { [92] }\end{array}$ & ESP & Catalonian Health Survey (CHS) & 1994 & $\begin{array}{l}\mathrm{n}=2866 \text { workers } \\
\text { and housewives }\end{array}$ & $25-64$ & all female & NP & Education & VLTPA \\
\hline $\begin{array}{l}\text { Bolívar et al., } 2010 \\
\text { [93] }\end{array}$ & ESP & Andalusia Health Survey & 1999, 2003 & $n=13193$ & $16+$ & $49 \%$ & NP & $\begin{array}{l}\text { Education Social class } \\
\text { Neighb. SEP }\end{array}$ & TLTPA \\
\hline $\begin{array}{l}\text { Borrell et al., 2000a } \\
\text { [94] }\end{array}$ & ESP & $\begin{array}{l}\text { Barcelona Health Interview } \\
\text { Survey }\end{array}$ & 1992 & $n=4171$ & $14+$ & $47 \%$ & $91 \%$ & Occupation & TPA VLTPA \\
\hline \multirow{3}{*}{$\begin{array}{l}\text { Borrell et al., 2000b } \\
\text { [95] }\end{array}$} & \multirow[t]{3}{*}{ ESP } & \multirow{3}{*}{$\begin{array}{l}\text { Barcelona Health Interview } \\
\text { Survey }\end{array}$} & 1986 & n $1986=7907$ & \multirow[t]{3}{*}{$14+$} & 1986: $46 \%$ & \multirow[t]{3}{*}{$88-93 \%$} & \multirow[t]{3}{*}{ Occupation } & \multirow[t]{3}{*}{ TPA } \\
\hline & & & 1992 & n $1992=5004$ & & 1992: 47\% & & & \\
\hline & & & 1994 & n $1994=2155$ & & 1994: 44\% & & & \\
\hline $\begin{array}{l}\text { De Vogli et al., } 2005 \\
\text { [96] }\end{array}$ & ITA & $\begin{array}{l}\text { Health Determinants Surveillance } \\
\text { System (HDSS) Survey }\end{array}$ & 2003 & $n=3327$ & $18-91$ & $52 \%$ & $57 \%$ & Social class & TLTPA \\
\hline Gal et al., 2005 [97] & PRT & Porto health survey & NP & $n=2004$ & $18+$ & $39 \%$ & $70 \%$ & Education Social class & TPA TLTPA \\
\hline $\begin{array}{l}\text { Lera-López \& Rapún- } \\
\text { Gárate, } 2005 \text { [98] }\end{array}$ & ESP & $\begin{array}{l}\text { Sport participation and consumer } \\
\text { expenditure in Navarra, Spain }\end{array}$ & 2004 & $n=700$ & $16-65$ & NP & NP & Income Education & VLTPA \\
\hline $\begin{array}{l}\text { Meseguer et al., } \\
2009 \text { [99] }\end{array}$ & ESP & $\begin{array}{l}\text { Non-communicable Disease Risk } \\
\text { Factor Surveillance System } \\
\text { (NCDRFSS) }\end{array}$ & $2000-05$ & $n=12037$ & $18-64$ & $49 \%$ & $65 \%$ & Education & TLTPA \\
\hline $\begin{array}{l}\text { Panagiotakos et al., } \\
\text { 2008a [100] }\end{array}$ & GRC & ATTICA study & 2001-02 & $n=3042$ & $18+$ & $50 \%$ & $75 \%$ & Education & TPA \\
\hline $\begin{array}{l}\text { Panagiotakos et al., } \\
\text { 2008b [101] }\end{array}$ & GRC & ATTICA study & 2001-02 & $n=3042$ & $18+$ & $50 \%$ & $75 \%$ & Education & TPA \\
\hline $\begin{array}{l}\text { Pascual et al., } 2007 \\
{[102]}\end{array}$ & ESP & Spanish Health Study & 2001 & $n=19324$ & $16-74$ & $49 \%$ & $85 \%$ & $\begin{array}{l}\text { Income Education } \\
\text { Social class Neighb. } \\
\text { SEP }\end{array}$ & TLTPA \\
\hline $\begin{array}{l}\text { Pascual et al., } 2009 \\
{[103]}\end{array}$ & ESP & $\begin{array}{l}\text { General survey on customs } \\
\text { regarding media and leisure } \\
\text { activities }\end{array}$ & 1999 & $n=25982$ & $25-74$ & $49 \%$ & $70 \%$ & $\begin{array}{l}\text { Income Education } \\
\text { Neighb. SEP }\end{array}$ & VLTPA \\
\hline $\begin{array}{l}\text { Pitsavos et al., } 2005 \\
\text { [104] }\end{array}$ & GRC & ATTICA study & 2001-02 & $n=3042$ & $20-89$ & $50 \%$ & $75 \%$ & $\begin{array}{l}\text { Income Education } \\
\text { Social class }\end{array}$ & VLTPA \\
\hline $\begin{array}{l}\text { Santos et al., } 2009 \\
\text { [105] }\end{array}$ & PRT & $\begin{array}{l}\text { Azorean Physical Activity and } \\
\text { Health Study }\end{array}$ & 2004 & $n=9991$ & $18-65$ & $43 \%$ & $88 \%$ & Income Education & TPA \\
\hline $\begin{array}{l}\text { Schröder et al., } 2004 \\
\text { [106] }\end{array}$ & ESP & $\begin{array}{l}\text { Gerona cardiovascular risk } \\
\text { factor and lifestyle study }\end{array}$ & 1994-96 & $n=1748$ & $25-74$ & $48 \%$ & $73 \%$ & Education & TLTPA VLTPA \\
\hline \multicolumn{10}{|l|}{$\begin{array}{l}\text { Scandinavian } \\
\text { region }\end{array}$} \\
\hline & SWE & & 2000 & & $18-64$ & $56 \%$ & $59 \%$ & Education & TLTPA \\
\hline
\end{tabular}


Table 1 Characteristics of the 131 included studies ordered by European region (Continued)

\begin{tabular}{|c|c|c|c|c|c|c|c|c|c|}
\hline $\begin{array}{l}\text { Ali \& Lindström, } \\
2006 \text { [107] }\end{array}$ & & $\begin{array}{l}2000 \text { public health survey in } \\
\text { Scania }\end{array}$ & & $\begin{array}{l}\mathrm{n}=5180 \text { workforce } \\
\text { or unemployed }\end{array}$ & & & & & \\
\hline \multirow[t]{3}{*}{$\begin{array}{l}\text { Andersen et al., } 2000 \\
\text { [108] }\end{array}$} & \multirow[t]{3}{*}{ DNK } & $\begin{array}{l}\text { Copenhagen City Heart Study } \\
\text { (CCHS) }\end{array}$ & \multirow[t]{3}{*}{ 1964-92 } & \multirow[t]{3}{*}{$n=30640$} & \multirow[t]{3}{*}{$20-93$} & \multirow[t]{3}{*}{$56 \%$} & \multirow[t]{3}{*}{$69-95 \%$} & \multirow[t]{3}{*}{ Education } & \multirow[t]{3}{*}{$\begin{array}{l}\text { TLTPA VLTPA } \\
\text { AT }\end{array}$} \\
\hline & & Copenhagen Male Study (CMS) & & & & & & & \\
\hline & & $\begin{array}{l}\text { Glostrup Population Study (GPS) } \\
\text { (pooled) }\end{array}$ & & & & & & & \\
\hline $\begin{array}{l}\text { Barengo et al., } 2006 \\
\text { [109] }\end{array}$ & FIN & National FINRISK Study & $1972-97$ & $n=33712$ & $30-59$ & $49 \%$ & $71-95 \%$ & Education Social class & TPA \\
\hline $\begin{array}{l}\text { Bergman et al., } 2008 \\
{[110]}\end{array}$ & SWE & $\begin{array}{l}\text { International Prevalence Study } \\
\text { (IPS) Sweden }\end{array}$ & 2003 & $n=1470$ & $18-74$ & $47 \%$ & $59 \%$ & Income Education & TPA \\
\hline $\begin{array}{l}\text { Borodulin et al., } 2008 \\
\text { [111] }\end{array}$ & FIN & National FINRISK Study & 2002 & $n=4437$ & $25-64$ & $44 \%$ & $59-70 \%$ & Education & TLTPA \\
\hline $\begin{array}{l}\text { Cubbin et al., } 2006 \\
\text { [112] }\end{array}$ & SWE & $\begin{array}{l}\text { Swedish Annual Level of Living } \\
\text { Survey }\end{array}$ & $1996-2000$ & $n=10890$ & $25-64$ & $49 \%$ & $80 \%$ & $\begin{array}{l}\text { Individual SEP Neighb. } \\
\text { SEP }\end{array}$ & VLTPA \\
\hline $\begin{array}{l}\text { Engström, } 2008 \\
\text { [113] }\end{array}$ & SWE & Sport Habitus Study Sweden & 2007 & $n=1518$ & 53 & NP & $77 \%$ & Education & VLTPA \\
\hline $\begin{array}{l}\text { Häkkinen et al., } 2006 \\
\text { [114] }\end{array}$ & FIN & $\begin{array}{l}\text { Northern Finland } 1966 \text { Birth } \\
\text { Cohort }\end{array}$ & 1998 & $n=4343$ & 31 & $46 \%$ & $76 \%$ & Education & TLTPA \\
\hline \multirow{5}{*}{$\begin{array}{l}\text { Henriksson et al., } \\
2003[115]\end{array}$} & \multirow[t]{5}{*}{ SWE } & \multirow{5}{*}{$\begin{array}{l}\text { Cardiovascular Risk Factor Study } \\
\text { in Southern Sweden (CRISS) }\end{array}$} & 1990 & n $1990=$ & 37 & \multirow[t]{5}{*}{$100 \%$} & 1990: & \multirow[t]{5}{*}{ Education } & \multirow[t]{5}{*}{ TLTPA } \\
\hline & & & 1993 & 991 & 40 & & $68 \%$ & & \\
\hline & & & 1996 & n $1993=770$ & 43 & & $\begin{array}{l}\text { 1993: } 78 \% \\
*\end{array}$ & & \\
\hline & & & & n $1996=702$ & & & $\begin{array}{l}\text { 1996: } 71 \% \\
*\end{array}$ & & \\
\hline & & & & & & & $\begin{array}{l}\text { *of } \\
\text { baseline }\end{array}$ & & \\
\hline Hu et al., 200 [37] & FIN & National FINRISK Study & $\begin{array}{l}1982,1987 \\
1992\end{array}$ & $n=14290$ & $35-64$ & $48 \%$ & $74-88 \%$ & Education & OPA TLTPA AT \\
\hline $\begin{array}{l}\text { Kivimäki et al., } 2007 \\
\text { [116] }\end{array}$ & FIN & Finnish Public Sector Study & 2000-02 & $n=48592$ & $17-65$ & $19 \%$ & $68 \%$ & Individual SEP & TPA \\
\hline $\begin{array}{l}\text { Korniloff et al., } 2010 \\
\text { [117] }\end{array}$ & FIN & $\begin{array}{l}\text { Finnish type } 2 \text { diabetes (FIN-D2D) } \\
\text { survey }\end{array}$ & 2007 & $n=2778$ & $45-74$ & $47 \%$ & $64 \%$ & Income Education & TLTPA \\
\hline $\begin{array}{l}\text { Laaksonen et al., } \\
2002[118]\end{array}$ & FIN & $\begin{array}{l}\text { Finnish Adult Health Behaviour } \\
\text { Survey }\end{array}$ & $1991-98$ & $\begin{array}{l}\mathrm{n}=26014 \text { civil } \\
\text { servants }\end{array}$ & $15-64$ & $47 \%$ & $69-76 \%$ & Education & TLTPA \\
\hline $\begin{array}{l}\text { Laaksonen et al., } \\
2008 \text { [119] }\end{array}$ & FIN & $\begin{array}{l}\text { Finnish Adult Health Behaviour } \\
\text { Survey }\end{array}$ & 1979-2001 & $n=60608$ & $25-64$ & $48 \%$ & $62-86 \%$ & Education & TLTPA \\
\hline \multirow[t]{2}{*}{$\begin{array}{l}\text { Lagerros et al., } 2009 \\
\text { [120] }\end{array}$} & SWE & $\begin{array}{l}\text { The Swedish National March } \\
\text { Cohort }\end{array}$ & 1997 & $n=42150$ & $18-94$ & $36 \%$ & NP & Education & TPA \\
\hline & SWE & & 2006 & $n=6966$ & $18-84$ & $45 \%$ & $54 \%$ & & TPA \\
\hline
\end{tabular}


Table 1 Characteristics of the 131 included studies ordered by European region (Continued)

\begin{tabular}{|c|c|c|c|c|c|c|c|c|c|}
\hline $\begin{array}{l}\text { Leijon et al., } 2010 \\
{[121]}\end{array}$ & & $\begin{array}{l}\text { Public Health Survey Ostergotland } \\
\text { County }\end{array}$ & & & & & & $\begin{array}{l}\text { Education Self-reported } \\
\text { economy }\end{array}$ & \\
\hline $\begin{array}{l}\text { Lindström et al., } \\
2001 \text { [122] }\end{array}$ & SWE & $\begin{array}{l}\text { The Malmö Diet and Cancer } \\
\text { Study }\end{array}$ & 1992-94 & $\mathrm{n}=11837$ & $45-65$ & $45 \%$ & $39 \%$ & Social class & TLTPA \\
\hline $\begin{array}{l}\text { Lindström et al., } \\
\text { 2003a [123] }\end{array}$ & SWE & $\begin{array}{l}\text { The Malmö Public Health } \\
\text { Survey }\end{array}$ & 1986, 1994 & $n=3861$ & $21-81$ & $47 \%$ & $71-74 \%$ & Education & TLTPA \\
\hline $\begin{array}{l}\text { Lindström et al., } \\
\text { 2003b [124] }\end{array}$ & SWE & $\begin{array}{l}\text { The Malmö Public Health } \\
\text { Survey }\end{array}$ & 1994 & $n=3377$ & $20-80$ & NP & $71 \%$ & Education & TLTPA \\
\hline $\begin{array}{l}\text { Mäkinen et al., } 2009 \\
{[125]}\end{array}$ & FIN & $\begin{array}{l}\text { Finnish Adult Health Behaviour } \\
\text { Survey }\end{array}$ & 1978-2002 & $\begin{array}{l}\mathrm{n}=50815 \\
\text { employed }\end{array}$ & $25-64$ & $50 \%$ & $62-86 \%$ & $\begin{array}{l}\text { Income Education } \\
\text { Social class }\end{array}$ & TLTPA AT \\
\hline $\begin{array}{l}\text { Mäkinen et al., 2010a } \\
\text { [126] }\end{array}$ & FIN & The Health 2000 Survey & 2000-01 & $\mathrm{n}=3355$ employed & $30+$ & $46 \%$ & $85-89 \%$ & Social class & OPA TLTPA \\
\hline $\begin{array}{l}\text { Mäkinen et al., } \\
\text { 2010b [127] }\end{array}$ & FIN & The Health 2000 Survey & 2000-01 & $n=7112$ & $30+$ & $45 \%$ & $84-89 \%$ & $\begin{array}{l}\text { Income Education } \\
\text { Social class }\end{array}$ & TLTPA \\
\hline $\begin{array}{l}\text { Mäkinen et al., 2010c } \\
{[128]}\end{array}$ & FIN & National FINRISK study & 2002 & $n=4408$ & $25-64$ & $44 \%$ & $60-70 \%$ & Education & TLTPA \\
\hline Molarius, 2003 [129] & SWE & Varmland County Survey & 2000 & $n=6394$ & $25-74$ & $47 \%$ & $70 \%$ & Education & TLTPA \\
\hline $\begin{array}{l}\text { Nielsen et al., } 2006 \\
\text { [130] }\end{array}$ & DNK & Odense Androgen Study & 2002,2003 & $n=783$ & $20-29$ & $100 \%$ & $73 \%$ & Education & TLTPA \\
\hline $\begin{array}{l}\text { Norman et al., } 2002 \\
\text { [131] }\end{array}$ & SWE & COSM (cohort of Swedish men) & 1997 & $n=33466$ & $45-79$ & $100 \%$ & $48 \%$ & Education & TPA TLTPA \\
\hline $\begin{array}{l}\text { Novak et al., } 2006 \\
\text { [132] }\end{array}$ & SWE & Swedish Cohort Study & 1981, 1995 & $n=1044$ & 16,30 & $52 \%$ & $96 \%$ & Education & TPA \\
\hline $\begin{array}{l}\text { Orsini et al., } 2007 \\
\text { [133] }\end{array}$ & SWE & $\begin{array}{l}\text { Swedish Mammography Study } \\
\text { (SMC97) }\end{array}$ & 1997 & $\mathrm{n}=38988$ & $40-75$ & all female & $70 \%$ & Education & TPA \\
\hline $\begin{array}{l}\text { Osler et al., } 2000 \\
\text { [134] }\end{array}$ & DNK & MONICA - Denmark & $\begin{array}{l}\text { 1982-1984, } \\
\text { 1987, 1991-92 }\end{array}$ & $n=6695$ & $\begin{array}{l}30,40 \\
50,60\end{array}$ & $50 \%$ & $73-79 \%$ & Education & TLTPA \\
\hline $\begin{array}{l}\text { Osler et al., } 2001 \\
\text { [135] }\end{array}$ & DNK & $\begin{array}{l}\text { Children of the Copenhagen City } \\
\text { Heart Study }\end{array}$ & 1992 & $n=317$ & $19-31$ & $51 \%$ & $52 \%$ & Education & TLTPA \\
\hline $\begin{array}{l}\text { Osler et al., } 2008 \\
\text { [136] }\end{array}$ & DNK & Metropolit cohort (1965) & 2004 & $n=6292$ & 51 & $100 \%$ & $66 \%$ & Education & TLTPA \\
\hline \multirow{4}{*}{$\begin{array}{l}\text { Petersen et al., } 2010 \\
\text { [137] }\end{array}$} & \multirow[t]{4}{*}{ DNK } & \multirow{4}{*}{$\begin{array}{l}\text { Danish National Health Interview } \\
\text { Survey }\end{array}$} & 1987 & n $1987=4752$ & \multirow[t]{4}{*}{$16+$} & \multirow[t]{4}{*}{$49 \%$} & 1987: $80 \%$ & \multirow[t]{4}{*}{ Education } & \multirow[t]{4}{*}{ TLTPA } \\
\hline & & & 1994 & n $1994=4667$ & & & 1994: $78 \%$ & & \\
\hline & & & 2000 & n $2000=16688$ & & & 2000: $74 \%$ & & \\
\hline & & & 2005 & n $2005=14566$ & & & 2005: 67\% & & \\
\hline Piro et al., 2007 [138] & NOR & Oslo Health Study (HUBRO) & 2000 & $n=14608$ & $\begin{array}{l}30,40 \\
45,60\end{array}$ & $45 \%$ & $46 \%$ & $\begin{array}{l}\text { Income Education } \\
\text { Neighb. SEP }\end{array}$ & VLTPA \\
\hline $\begin{array}{l}\text { Pudaric et al., } 2000 \\
\text { [139] }\end{array}$ & SWE & Migrants in Sweden Study & 1988-89 & $n=3100$ & $55-74$ & $47 \%$ & $80 \%$ & Income & TPA \\
\hline
\end{tabular}


Table 1 Characteristics of the 131 included studies ordered by European region (Continued)

\begin{tabular}{|c|c|c|c|c|c|c|c|c|c|}
\hline $\begin{array}{l}\text { Pulkki et al., 2003a } \\
\text { [140] }\end{array}$ & FIN & $\begin{array}{l}\text { Cardiovascular Risks in Young Finns } \\
\text { (CRYF) study }\end{array}$ & 1983, 1992 & $n=1219$ & $\begin{array}{l}12-21 \\
21-30\end{array}$ & $44 \%$ & $62 \%$ & Individual SEP & TLTPA \\
\hline $\begin{array}{l}\text { Pulkki et al., 2003b } \\
\text { [141] }\end{array}$ & FIN & $\begin{array}{l}\text { Cardiovascular Risks in Young Finns } \\
\text { (CRYF) study }\end{array}$ & 1983, 1992 & $n=1125$ & $\begin{array}{l}12-21 \\
21-30\end{array}$ & $58 \%$ & $57 \%$ & Education & TLTPA \\
\hline $\begin{array}{l}\text { Salonen et al., } 2010 \\
\text { [142] }\end{array}$ & FIN & $\begin{array}{l}\text { Sub-study of the Helsinki Birth } \\
\text { Cohort Study }\end{array}$ & 2001-04 & $n=1967$ & $57-71$ & $46 \%$ & NP & Education Social class & TLTPA \\
\hline \multirow[t]{3}{*}{$\begin{array}{l}\text { Schnohr et al., } 2004 \\
\text { [143] }\end{array}$} & \multirow[t]{3}{*}{ DNK } & $\begin{array}{l}\text { Copenhagen City Heart Study } \\
\text { (CCHS) }\end{array}$ & \multirow[t]{3}{*}{$1967-86$} & \multirow[t]{3}{*}{$n=30635$} & \multirow[t]{3}{*}{$20-93$} & \multirow[t]{3}{*}{$53 \%$} & \multirow[t]{3}{*}{ NP } & \multirow[t]{3}{*}{ Education } & \multirow[t]{3}{*}{ TLTPA } \\
\hline & & Copenhagen Male Study (CMS) & & & & & & & \\
\hline & & $\begin{array}{l}\text { Glostrup Population Study (GPS) } \\
\text { (pooled) }\end{array}$ & & & & & & & \\
\hline $\begin{array}{l}\text { Simonen et al., } 2003 \\
\text { [144] }\end{array}$ & FIN & Finnish Twin Cohort & 1975,1981 & $\begin{array}{l}\mathrm{n}=224 \\
\text { monozygotic twins }\end{array}$ & $35-69$ & NP & $82 \%$ & Education & VLTPA \\
\hline $\begin{array}{l}\text { Sjögren \& } \\
\text { Stjernberg, } 2010 \\
\text { [145] }\end{array}$ & SWE & $\begin{array}{l}\text { Swedish National Study on } \\
\text { Aging and Care (SNAC) }\end{array}$ & 2001-03 & $\mathrm{n}=999$ & $60-96$ & $45 \%$ & $61 \%$ & Education & TLTPA \\
\hline $\begin{array}{l}\text { Strand \& Tverdal, } \\
2004 \text { [146] }\end{array}$ & NOR & $\begin{array}{l}\text { Cardiovascular disease study in } \\
\text { Norway }\end{array}$ & 1970 & $n=44684$ & $35-49$ & $51 \%$ & $91 \%$ & Education & TLTPA \\
\hline $\begin{array}{l}\text { Strandhagen et al., } \\
2010 \text { [147] }\end{array}$ & SWE & $\begin{array}{l}\text { The INTERGENE research } \\
\text { programme }\end{array}$ & 2001-04 & $n=3581$ & $25-74$ & $47 \%$ & $42 \%$ & Education & TLTPA \\
\hline $\begin{array}{l}\text { Suadicani et al., } 2001 \\
\text { [42] }\end{array}$ & DNK & Copenhagen Male Study & 1970-71 & $n=5028$ & $40-59$ & $100 \%$ & $87 \%$ & Social class & OPA TLTPA \\
\hline $\begin{array}{l}\text { Suadicani et al., } 2005 \\
\text { [148] }\end{array}$ & DNK & Copenhagen Male Study & $\begin{array}{l}1970-71 \\
1985-86\end{array}$ & $n=3290$ & $40-74$ & $100 \%$ & $75-87 \%$ & Social class & TLTPA \\
\hline $\begin{array}{l}\text { Tammelin et al., } \\
2003 \text { [149] }\end{array}$ & FIN & $\begin{array}{l}\text { Northern Finland } 1966 \text { Birth } \\
\text { Cohort }\end{array}$ & 1998 & $n=7794$ & 31 & $46 \%$ & $75 \%$ & Education & TLTPA \\
\hline $\begin{array}{l}\text { Wang et al., } 2010 \\
\text { [34] }\end{array}$ & FIN & $\begin{array}{l}\text { National FINRISK Study } \\
\text { (pooled data) }\end{array}$ & $\begin{array}{l}\text { 1972, 1977, } \\
1982,1987 \\
1992,1997, \\
2002\end{array}$ & $\mathrm{n}=58208$ & $24-74$ & $49 \%$ & $65-88 \%$ & Education & OPA TLTPA AT \\
\hline $\begin{array}{l}\text { Wemme \& Rosvall, } \\
2005 \text { [150] }\end{array}$ & SWE & Scania Health Survey & 1999-2000 & $\mathrm{n}=7169$ employed & NP & $54 \%$ & $59 \%$ & Education Social class & TLTPA \\
\hline \multicolumn{10}{|l|}{$\begin{array}{l}\text { Anglo-Saxon } \\
\text { region }\end{array}$} \\
\hline Adams, 2009 [151] & GBR & $\begin{array}{l}\text { English Longitudinal Study of } \\
\text { Ageing (ELSA) }\end{array}$ & 2002 & $n=10864$ & $50+$ & $47 \%$ & NP & Education & TPA \\
\hline Adams, 2010 [29] & GBR & $\begin{array}{l}2005 \text { UK Time Use Survey } \\
\text { (part of National Statistics } \\
\text { Omnibus Survey) }\end{array}$ & 2005 & $n=3933$ & $16+$ & $48 \%$ & $49 \%$ & Education Social class & AT \\
\hline $\begin{array}{l}\text { Allender et al., } 2008 \\
\text { [15] }\end{array}$ & GBR & Health Survey for England & 2003 & $n=13974$ & $16+$ & $45 \%$ & $66 \%$ & Education Social class & TPA TLTPA \\
\hline
\end{tabular}


Table 1 Characteristics of the 131 included studies ordered by European region (Continued)

\begin{tabular}{|c|c|c|c|c|c|c|c|c|c|}
\hline $\begin{array}{l}\text { Amuzu et al., } 2009 \\
\text { [152] }\end{array}$ & GBR & $\begin{array}{l}\text { British Women's Heart and Health } \\
\text { Study }\end{array}$ & $1999-2001$ & $n=3522$ & $60-79$ & all female & NP & $\begin{array}{l}\text { Individual SEP Neighb. } \\
\text { SEP }\end{array}$ & TPA \\
\hline \multirow{2}{*}{$\begin{array}{l}\text { Bartley et al., } 2000 \\
\text { [153] }\end{array}$} & \multirow[t]{2}{*}{ GBR } & Health and Lifestyle study (HALS) & 1984 & n $1984=2176$ & \multirow[t]{2}{*}{$20-64$} & \multirow[t]{2}{*}{$100 \%$} & \multirow[t]{2}{*}{ NP } & \multirow[t]{2}{*}{ Social class } & \multirow[t]{2}{*}{ VLTPA } \\
\hline & & Health Survey for England (HSfE) & 1993 & n $1993=4723$ & & & & & \\
\hline $\begin{array}{l}\text { Bartley et al., } 2004 \\
{[154]}\end{array}$ & GBR & Whitehall II Study & $1985-88$ & $\begin{array}{l}\mathrm{n}=5458 \text { civil } \\
\text { servants }\end{array}$ & $35-55$ & $74 \%$ & $73 \%$ & Social class & TLTPA \\
\hline $\begin{array}{l}\text { Chaudhury \& } \\
\text { Shelton, } 2010 \text { [155] }\end{array}$ & GBR & Health Survey for England (HSfE) & 2006 & $n=1550$ & $60-69$ & $46 \%$ & NP & $\begin{array}{l}\text { Income Social class } \\
\text { Neighb. SEP }\end{array}$ & TPA \\
\hline $\begin{array}{l}\text { Ecob \& Macintyre, } \\
2000[156]\end{array}$ & GBR & West of Scotland 20-07 Study & 1987, 1988 & $n=3036$ & $\begin{array}{l}15,35 \\
55\end{array}$ & NP & NP & Neighb. SEP & VLTPA \\
\hline $\begin{array}{l}\text { Harrison et al., } 2006 \\
\text { [157] }\end{array}$ & GBR & $\begin{array}{l}\text { Physical activity in North-West } \\
\text { England }\end{array}$ & 2001 & $n=15465$ & $18+$ & $45 \%$ & $70 \%$ & $\begin{array}{l}\text { Neighb. SEP Home } \\
\text { owner }\end{array}$ & TPA \\
\hline $\begin{array}{l}\text { Heslop et al., } 2001 \\
\text { [158] }\end{array}$ & GBR & $\begin{array}{l}\text { Cohort of workers recruited } \\
\text { from workplaces in Western } \\
\text { Scotland between } 1970 \text { and } 1973\end{array}$ & $1970-73$ & $\mathrm{n}=958$ employed & $\begin{array}{l}\text { working } \\
\text { age }\end{array}$ & all female & $70 \%$ & $\begin{array}{l}\text { Education Social class } \\
\text { Neighb. SEP }\end{array}$ & TLTPA \\
\hline $\begin{array}{l}\text { Hillsdon et al., } 2008 \\
\text { [159] }\end{array}$ & GBR & $\begin{array}{l}\text { British Women's Heart and } \\
\text { Health Study }\end{array}$ & 1999-2001 & $n=4286$ & $60-79$ & all female & NP & $\begin{array}{l}\text { Individual SEP Neighb. } \\
\text { SEP }\end{array}$ & TPA \\
\hline \multirow[t]{5}{*}{$\begin{array}{l}\text { Lahelma et al., } 2010 \\
\text { [160] }\end{array}$} & \multirow[t]{5}{*}{ GBR FIN } & \multirow[t]{2}{*}{$\begin{array}{l}\text { The London-based Whitehall II } \\
\text { study (WHII) }\end{array}$} & \multirow[t]{2}{*}{ WHII: 1997-99 } & \multirow[t]{2}{*}{ n WHII $=2678$} & \multirow[t]{2}{*}{$\begin{array}{l}\text { WHII: } \\
45-\end{array}$} & \multirow[t]{2}{*}{$\begin{array}{l}\text { WHIII: } 76 \% \\
\text { HHS: }\end{array}$} & $\begin{array}{l}\text { WHII: } 73 \% \\
\text { HHS: }\end{array}$ & \multirow[t]{2}{*}{ Social class } & \multirow[t]{2}{*}{ TLTPA } \\
\hline & & & & & & & $67 \%$ & & \\
\hline & & \multirow[t]{3}{*}{ The Helsinki Health Study (HHS) } & & & & \multirow[t]{3}{*}{$17 \%$} & & & \\
\hline & & & HHS: 2001-02 & $\mathrm{n} \mathrm{HHS}=8960$ & 60 & & & & \\
\hline & & & & $\begin{array}{l}\text { white collar } \\
\text { employees }\end{array}$ & $\begin{array}{l}\text { HHS: } \\
40-60\end{array}$ & & & & \\
\hline $\begin{array}{l}\text { Livingstone et al., } \\
2001 \text { [161] }\end{array}$ & $\mathrm{IRL}$ & $\begin{array}{l}\text { North/South Ireland Food } \\
\text { Consumption Survey (NSIFCS) }\end{array}$ & $1997-99$ & $n=1379$ & $18-64$ & $48 \%$ & NP & Social class & VLTPA \\
\hline Lunn, 2010 [162] & $\mathrm{IRL}$ & $\begin{array}{l}\text { The Survey of Sport and Physical } \\
\text { Exercise }\end{array}$ & 2003 & $n=2896$ & $18+$ & NP & $67 \%$ & Income Education & VLTPA \\
\hline \multirow{2}{*}{$\begin{array}{l}\text { Mein et al., } 2005 \\
\text { [163] }\end{array}$} & \multirow[t]{2}{*}{ GBR } & \multirow[t]{2}{*}{ Whitehall II study } & \multirow[t]{2}{*}{$1997-99$} & $n=6224$ & \multirow[t]{2}{*}{$45-69$} & \multirow[t]{2}{*}{$72 \%$} & \multirow[t]{2}{*}{$71 \%$} & \multirow[t]{2}{*}{ Social class } & \multirow[t]{2}{*}{ TLTPA } \\
\hline & & & & civil servants & & & & & \\
\hline $\begin{array}{l}\text { Mullineaux et al., } \\
2001 \text { [164] }\end{array}$ & GBR & $\begin{array}{l}\text { Allied Dunbar National Fitness } \\
\text { Survey of English Adults (ADNFS) }\end{array}$ & 1990 & $n=2005$ & $16+$ & NP & NP & Education & TPA \\
\hline $\begin{array}{l}\text { Mutrie \& Hannah, } \\
2004 \text { [165] }\end{array}$ & GBR & $\begin{array}{l}\text { West of Scotland Twenty-07 } \\
\text { study ( } 3^{\text {rd }} \text { wave) }\end{array}$ & $1995-96$ & $n=2153$ & $\begin{array}{l}24,44 \\
64\end{array}$ & $42 \%$ & NP & Social class & OPA TLTPA \\
\hline $\begin{array}{l}\text { Myint et al., } 2006 \\
{[166]}\end{array}$ & GBR & EPIC study & $1993-97$ & $n=23085$ & $40-79$ & $46 \%$ & NP & Social class & TPA \\
\hline \multirow{2}{*}{$\begin{array}{l}\text { Poortinga, } 2007 \\
\text { [167] }\end{array}$} & GBR & Health Survey for England & 2003 & $n=11617$ & $16-64$ & NP & NP & Social class & OPA VLTPA \\
\hline & GBR & British Household Panel Survey & & $n=9473$ & $18-64$ & $48 \%$ & $74 \%$ & & TLTPA \\
\hline
\end{tabular}


Table 1 Characteristics of the 131 included studies ordered by European region (Continued)

\begin{tabular}{|c|c|c|c|c|c|c|c|c|c|}
\hline \multicolumn{3}{|l|}{$\begin{array}{l}\text { Popham \& Mitchell, } \\
2006 \text { [168] }\end{array}$} & \multicolumn{5}{|l|}{$\begin{array}{l}\text { 1996, 1998, } \\
2000,2002\end{array}$} & \multicolumn{2}{|l|}{$\begin{array}{l}\text { Income Education } \\
\text { Social class School } \\
\text { type (fee-paying) }\end{array}$} \\
\hline $\begin{array}{l}\text { Popham \& Mitchell, } \\
2007 \text { [16] }\end{array}$ & GBR & $\begin{array}{l}2003 \text { Scottish Health Survey } \\
\text { (SHS) }\end{array}$ & 2003 & $n=5287$ & $25-64$ & $44 \%$ & $60 \%$ & Individual SEP & $\begin{array}{l}\text { TPA OPA } \\
\text { VLTPA }\end{array}$ \\
\hline Popham, 2010 [169] & GBR & $\begin{array}{l}2003 \text { Scottish Health Survey } \\
\text { (SHS) }\end{array}$ & 2003 & $n=2770$ & $35-54$ & NP & $60 \%$ & Social class & VLTPA \\
\hline $\begin{array}{l}\text { Stamatakis \& } \\
\text { Chaudhury, } 2008 \\
\text { [170] }\end{array}$ & GBR & $\begin{array}{l}\text { Health Survey for England } \\
\text { (HSfE) }\end{array}$ & $\begin{array}{l}1997,1998, \\
2003,2004 \\
2006\end{array}$ & $n=60938$ & $16+$ & $45 \%$ & $61-71 \%$ & $\begin{array}{l}\text { Income Education } \\
\text { Social class }\end{array}$ & VLTPA \\
\hline \multirow{2}{*}{$\begin{array}{l}\text { Stringhini et al., } 2010 \\
\text { [3] }\end{array}$} & \multirow[t]{2}{*}{ GBR } & \multirow[t]{2}{*}{ Whitehall II cohort } & \multirow[t]{2}{*}{ 1985-88 } & $n=9590$ & \multirow[t]{2}{*}{$35-55$} & \multirow[t]{2}{*}{$68 \%$} & \multirow[t]{2}{*}{$73 \%$} & \multirow[t]{2}{*}{ Social class } & \multirow[t]{2}{*}{ TLTPA } \\
\hline & & & & civil servants & & & & & \\
\hline $\begin{array}{l}\text { Wardle \& Griffith, } \\
2001[171]\end{array}$ & GBR & British Omnibus Study & 1999 & $n=1790$ & $16+$ & $50 \%$ & $70 \%$ & Social class & VLTPA \\
\hline $\begin{array}{l}\text { Wardle \& Steptoe, } \\
2003 \text { [172] }\end{array}$ & GBR & British Omnibus Study & 2000 & $n=1691$ & $16+$ & $45 \%$ & $62 \%$ & Social class & VLTPA \\
\hline $\begin{array}{l}\text { Watt et al., } 2009 \\
{[173]}\end{array}$ & GBR & $\begin{array}{l}\text { British Women's Heart and } \\
\text { Health Study }\end{array}$ & 1999-2001 & $n=3523$ & $60-79$ & all female & NP & Individual SEP & TPA \\
\hline
\end{tabular}

${ }^{\mathrm{a}} \mathrm{EU}=$ European Union, ALB = Albania, BEL = Belgium, CZE = Czech Republic, DEU = Germany, $\mathrm{DNK}=$ Denmark, ESP $=$ Spain, EST $=$ Estonia, FIN $=$ Finland, FRA $=$ France, GBR $=$ United Kingdom, GRC = Greece, HRZ = Croatia (local name is Hrvatska), HUN = Hungary, IRL = Ireland, ITA = Italy, LTU = Lithuania, LVA = Latvia, NLD = The Netherlands, NOR = Norway, POL = Poland, PRT $=$ Portugal, SVN $=$ Slovenia, SWE $=$ Sweden, SWI = Switzerland.

${ }^{\mathrm{b}}$ Sample characteristics only provided when a specific subsample from the population was studied (e.g. working people, civil servants, etc.).

c NP = Not Provided.

${ }^{\mathrm{d}}$ SEP $=$ socioeconomic position, Neighb. $=$ neighbourhood, Individual SEP $=$ composite measure of different individual SEP indicators.

e PA = Physical Activity, TPA = Total Physical Activity, OPA = Occupational Physical Activity, TLTPA = Total Leisure-time Physical Activity, VLTPA = Vigorous Leisure-time Physical Activity, AT = Active Transport. 
the PA outcome of interest. A '0' means that there was no significant (linear) association found. Significance was judged with $\alpha=0.05$. When there were more than two categories, the overall test of significance, or trend test was used (when available). If not available, significance was judged by looking at the significance level of the difference between the two most extreme groups. When there was no trend, or a curvilinear trend, for example when only the middle group was significantly different (but not the extremes), the association was classified as being non significant. When the symbol is between brackets, no test of significance was reported and difference was judged solely on descriptive measures such as percentages.

When both adjusted and unadjusted results were presented in the manuscripts, the adjusted results were recorded into the table, including a notification of the variables that were used for adjustment. Duplicate articles on the same study population were only included in the tables if they contributed unique associations not previously reported. Distributions of reported positive, negative, and null associations were evaluated by gender, SEP indicator, and European region for each PA outcome (Tables 2 and 3).

\section{Quality assessment}

Since only observational studies were included in this study, methods for quality assessment were limited. Only a few basic quality guidelines were used as exclusion criteria. All included studies were treated equally in the results. To check if quality issues affected the results, sensitivity analyses were conducted for three common quality markers; response, adjustment, and sample size. In these analyses, the results were synthesized again after excluding the articles that did not report a response or studies with a response of less than 50\%. In separate analysis, associations that were not adjusted for at least age and gender were excluded from the results. Finally, the results were synthesized for those studies with at least 2000 participants. The results that were found in the subsets of associations were compared with the results obtained when all publications were included.

\section{Results}

The search strategy retrieved 7420 unique and potentially relevant titles (Figure 1). After scanning titles and abstracts a total of 193 articles were identified for inclusion. Sixtytwo articles were excluded, primarily because no association between SEP and PA was reported $(n=18)$, because of quality concerns $(n=11)$, because the population was older than $65(n=8)$, or because the study was conducted outside of Europe $(n=6)$. As a result, 131 studies were included in the current review.
These 131 studies reported on 105 study populations and 447 unique associations between a SEP indicator and PA outcome (Table 1). Most studies were conducted in Scandinavian countries and Great Britain. The majority of the sample sizes were large (e.g. including over 4000 participants) with a range from 224 to 60938 participants. In most studies the response was higher than 60\% (range 20$96 \%$ ) but approximately one quarter of the studies did not report any response percentage. Apart from the study by Van Dyck and colleagues [23] who used accelerometer data in addition to self-reported data, all studies relied on self-reported PA. The majority of the studies did not report the validity of the PA measure. The most frequently used validated PA questionnaire was the International PA Questionnaire (IPAQ) [24], other validated measures that were used were the Minnesota Leisure Time PA Questionnaire [25], the MONICA Optional Study of PA Questionnaire (MOSPA-Q) [26], the Short Questionnaire to Assess Health-Enhancing PA (SQUASH) [27], and the Modifiable Activity Questionnaire (MAQ) [26].

\section{Total physical activity}

There were 30 studies, with a total of 70 unique associations, which reported on the association between SEP IMDStril' total PA (Additional file 1, Table A1). Approximately equal amounts of positive $(n=28)$, null $(n=19)$ associations, and negative $(\mathrm{n}=23)$ associations were found (Table). This pattern did not differ between men and women. While most associations were not statistically significant with income as indicator of SEP, both positive and negative associations were found with education as indicator of SEP (Table 2). In Southern Europe, nine out of 12 assessed associations (75\%) indicated decreasing levels of physical activity by increasing levels of SEP, while in the Anglo-Saxon countries most (50\%) associations showed the opposite pattern (Table 3 ).

\section{Occupational physical activity}

There were 10 studies, with a total of 19 unique associations, which reported on the association between SEP and occupational PA (Additional file 1, Table A2). The majority of the associations (68\%) were negative, indicating that persons in lower socioeconomic groups did more occupational PA (Table 2). Patterns were similar for men and women. Almost all associations based on social class showed a negative relationship, while mixed patterns were found for education and income (Table 2). In studies in Eastern Europe, four out of six associations were non significant, while mainly negative associations were found in other regions of Europe (Table 3).

\section{Leisure-time physical activity}

Leisure-time PA was the most frequent domain of PA assessed in relation to SEP. A total of 112 studies reported 
Table 2 Distribution of positive, negative, and null associations by gender, SEP indicator, and PA domain ${ }^{\mathrm{a}}$

\begin{tabular}{|c|c|c|c|c|c|c|c|c|c|c|c|c|c|c|c|c|c|c|c|c|c|}
\hline \multirow{3}{*}{\multicolumn{2}{|c|}{ Physical activity ${ }^{b, c}$}} & \multirow{2}{*}{\multicolumn{4}{|c|}{$\begin{array}{r}\text { Total } \\
\text { TOTAL } \\
\end{array}$}} & \multicolumn{16}{|c|}{ Socioeconomic indicator } \\
\hline & & & & & & \multicolumn{4}{|c|}{ Income } & \multicolumn{4}{|c|}{ Education } & \multicolumn{4}{|c|}{ Social class } & \multicolumn{4}{|c|}{ Other } \\
\hline & & & + & 0 & - & & + & 0 & - & & + & 0 & - & & + & 0 & - & & + & 0 & - \\
\hline & Gender $^{d}$ & $\mathrm{n}$ & $\%$ & $\%$ & $\%$ & $\mathrm{n}$ & $\%$ & $\%$ & $\%$ & $\mathrm{n}$ & $\%$ & $\%$ & $\%$ & $\mathrm{n}$ & $\%$ & $\%$ & $\%$ & $\mathrm{n}$ & $\%$ & $\%$ & $\%$ \\
\hline \multirow[t]{3}{*}{ TPA } & $\pi$ & 34 & $41 \%$ & $24 \%$ & $35 \%$ & 5 & $20 \%$ & $60 \%$ & $20 \%$ & 16 & $50 \%$ & $6 \%$ & $44 \%$ & 6 & $17 \%$ & $17 \%$ & $67 \%$ & 7 & $57 \%$ & $43 \%$ & $0 \%$ \\
\hline & q & 36 & $39 \%$ & $31 \%$ & $31 \%$ & 5 & $0 \%$ & $80 \%$ & $20 \%$ & 16 & $38 \%$ & $25 \%$ & $38 \%$ & 6 & $17 \%$ & $33 \%$ & $50 \%$ & 9 & $78 \%$ & $11 \%$ & $11 \%$ \\
\hline & all & 70 & $40 \%$ & $27 \%$ & $33 \%$ & 10 & $10 \%$ & $70 \%$ & $20 \%$ & 32 & $44 \%$ & $16 \%$ & $41 \%$ & 12 & $17 \%$ & $\mathbf{2 5} \%$ & $58 \%$ & 16 & $69 \%$ & $25 \%$ & $6 \%$ \\
\hline \multirow[t]{3}{*}{ OPA } & $\pi$ & 10 & $10 \%$ & $20 \%$ & $70 \%$ & 1 & $0 \%$ & $100 \%$ & $0 \%$ & 4 & $25 \%$ & $25 \%$ & $50 \%$ & 4 & $0 \%$ & $0 \%$ & $100 \%$ & 1 & $0 \%$ & $0 \%$ & $100 \%$ \\
\hline & q & 9 & $11 \%$ & $33 \%$ & $56 \%$ & 1 & $0 \%$ & $100 \%$ & $0 \%$ & 4 & $25 \%$ & $25 \%$ & $50 \%$ & 3 & $0 \%$ & $33 \%$ & $67 \%$ & 1 & $0 \%$ & $0 \%$ & $100 \%$ \\
\hline & all & 19 & $11 \%$ & $26 \%$ & $63 \%$ & 2 & $0 \%$ & $100 \%$ & $0 \%$ & 8 & $\mathbf{2 5} \%$ & $25 \%$ & $50 \%$ & 7 & $0 \%$ & $14 \%$ & $86 \%$ & 2 & $0 \%$ & $0 \%$ & $100 \%$ \\
\hline \multirow[t]{3}{*}{ TLTPA } & $0^{\pi}$ & 104 & $68 \%$ & $31 \%$ & $1 \%$ & 17 & $71 \%$ & $29 \%$ & $0 \%$ & 56 & $68 \%$ & $30 \%$ & $2 \%$ & 19 & $79 \%$ & $21 \%$ & $0 \%$ & 12 & $50 \%$ & $50 \%$ & $0 \%$ \\
\hline & q & 96 & $68 \%$ & $32 \%$ & $0 \%$ & 17 & $47 \%$ & $53 \%$ & $0 \%$ & 49 & $78 \%$ & $22 \%$ & $0 \%$ & 19 & $68 \%$ & $32 \%$ & $0 \%$ & 11 & $55 \%$ & $45 \%$ & $0 \%$ \\
\hline & all & 200 & $68 \%$ & $32 \%$ & $1 \%$ & 34 & $59 \%$ & $41 \%$ & $0 \%$ & 105 & $72 \%$ & $27 \%$ & $1 \%$ & 38 & $74 \%$ & $26 \%$ & $0 \%$ & 23 & $52 \%$ & $48 \%$ & $0 \%$ \\
\hline \multirow[t]{3}{*}{ VLTPA } & 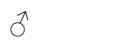 & 56 & $75 \%$ & $25 \%$ & $0 \%$ & 12 & $83 \%$ & $17 \%$ & $0 \%$ & 24 & $67 \%$ & $33 \%$ & $0 \%$ & 10 & $80 \%$ & $20 \%$ & $0 \%$ & 10 & $80 \%$ & $20 \%$ & $0 \%$ \\
\hline & q & 54 & $78 \%$ & $22 \%$ & $0 \%$ & 12 & $67 \%$ & $33 \%$ & $0 \%$ & 24 & $75 \%$ & $25 \%$ & $0 \%$ & 10 & $90 \%$ & $10 \%$ & $0 \%$ & 8 & $88 \%$ & $13 \%$ & $0 \%$ \\
\hline & all & 110 & $76 \%$ & $24 \%$ & $0 \%$ & 24 & $75 \%$ & $25 \%$ & $0 \%$ & 48 & $71 \%$ & $29 \%$ & $0 \%$ & 20 & $85 \%$ & $15 \%$ & $0 \%$ & 18 & $83 \%$ & $17 \%$ & $0 \%$ \\
\hline \multirow[t]{3}{*}{ AT } & $\pi$ & 26 & $35 \%$ & $31 \%$ & $35 \%$ & 4 & $25 \%$ & $25 \%$ & $50 \%$ & 14 & $50 \%$ & $36 \%$ & $14 \%$ & 3 & $33 \%$ & $33 \%$ & $33 \%$ & 5 & $0 \%$ & $20 \%$ & $80 \%$ \\
\hline & q & 22 & $41 \%$ & $27 \%$ & $32 \%$ & 4 & $50 \%$ & $0 \%$ & $50 \%$ & 12 & $58 \%$ & $25 \%$ & $17 \%$ & 3 & $0 \%$ & $67 \%$ & $33 \%$ & 3 & $0 \%$ & $33 \%$ & $67 \%$ \\
\hline & all & 48 & $38 \%$ & $29 \%$ & $33 \%$ & 8 & $38 \%$ & $13 \%$ & $50 \%$ & 26 & $\mathbf{5 4} \%$ & $\mathbf{3 1} \%$ & $15 \%$ & 6 & $17 \%$ & $50 \%$ & $33 \%$ & 8 & $0 \%$ & $25 \%$ & $75 \%$ \\
\hline
\end{tabular}

a SEP = socioeconomic position, $\mathrm{PA}=$ physical activity.

b TPA = Total Physical Activity, OPA = Occupational Physical Activity, TLTPA = Total Leisure-time Physical Activity, VLTPA = Vigorous Leisure-time Physical Activity, AT = Active Transport.

' The symbol ' + ' indicates the positive associations (a high SEP is associated with a high PA), the symbol ' - ' indicates negative associations (a high SEP is associated with a low PA), and ' $O$ ' indicates the neutral associations (no association found).

$\mathrm{d} \hat{d}=$ males, $q=$ females, all $=$ genders combined 
Table 3 Distribution of positive, negative, and null associations by gender, European region, and PA domain ${ }^{\text {a }}$

\begin{tabular}{|c|c|c|c|c|c|c|c|c|c|c|c|c|c|c|c|c|c|c|c|c|c|c|c|c|c|}
\hline \multirow{3}{*}{\multicolumn{2}{|c|}{ Physical activity ${ }^{b, c}$}} & \multicolumn{24}{|c|}{ European region } \\
\hline & & \multicolumn{4}{|c|}{ EU lackwide studies } & \multicolumn{4}{|c|}{ Western European region } & \multicolumn{4}{|c|}{ Eastern European region } & \multicolumn{4}{|c|}{ Southern European region } & \multicolumn{4}{|c|}{ Scandinavian region } & \multicolumn{4}{|c|}{ Anglo-Saxon region } \\
\hline & & & + & 0 & - & & + & 0 & - & & + & 0 & - & & + & 0 & - & & + & 0 & - & & + & 0 & - \\
\hline & Gender $^{d}$ & $\mathrm{n}$ & $\%$ & $\%$ & $\%$ & n & $\%$ & $\%$ & $\%$ & $\mathrm{n}$ & $\%$ & $\%$ & $\%$ & $\mathrm{n}$ & $\%$ & $\%$ & $\%$ & $\mathrm{n}$ & $\%$ & $\%$ & $\%$ & $\mathrm{n}$ & $\%$ & $\%$ & $\%$ \\
\hline \multirow[t]{3}{*}{ TPA } & 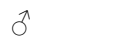 & 1 & $100 \%$ & $0 \%$ & $0 \%$ & 1 & $0 \%$ & $100 \%$ & $0 \%$ & 5 & $40 \%$ & $40 \%$ & $20 \%$ & 6 & $17 \%$ & $0 \%$ & $83 \%$ & 10 & $50 \%$ & $10 \%$ & $40 \%$ & 11 & $45 \%$ & $36 \%$ & $18 \%$ \\
\hline & q & 1 & $100 \%$ & $0 \%$ & $0 \%$ & 1 & $0 \%$ & $100 \%$ & $0 \%$ & 5 & $40 \%$ & $20 \%$ & $40 \%$ & 6 & $17 \%$ & $17 \%$ & $67 \%$ & 10 & $30 \%$ & $40 \%$ & $30 \%$ & 13 & $54 \%$ & $31 \%$ & $15 \%$ \\
\hline & all & 2 & $100 \%$ & $0 \%$ & $0 \%$ & 2 & $0 \%$ & $100 \%$ & $0 \%$ & 10 & $40 \%$ & $30 \%$ & $30 \%$ & 12 & $17 \%$ & $\mathbf{8} \%$ & $75 \%$ & 20 & $40 \%$ & $25 \%$ & $35 \%$ & 24 & $50 \%$ & $33 \%$ & $17 \%$ \\
\hline \multirow[t]{3}{*}{ OPA } & $\pi$ & 1 & $100 \%$ & $0 \%$ & $0 \%$ & - & - & - & - & 3 & $0 \%$ & $67 \%$ & $33 \%$ & - & - & - & - & 3 & $0 \%$ & $0 \%$ & $100 \%$ & 3 & $0 \%$ & $0 \%$ & $100 \%$ \\
\hline & q & 1 & $100 \%$ & $0 \%$ & $0 \%$ & - & - & - & - & 3 & $0 \%$ & $67 \%$ & $33 \%$ & - & - & - & - & 2 & $0 \%$ & $0 \%$ & $100 \%$ & 3 & $0 \%$ & $33 \%$ & $67 \%$ \\
\hline & all & 2 & $100 \%$ & $0 \%$ & $0 \%$ & - & - & - & - & 6 & $0 \%$ & $67 \%$ & $33 \%$ & - & - & - & - & 5 & $0 \%$ & $0 \%$ & $100 \%$ & 6 & $0 \%$ & $17 \%$ & $83 \%$ \\
\hline \multirow[t]{3}{*}{ TLTPA } & 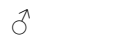 & 3 & $100 \%$ & $0 \%$ & $0 \%$ & 20 & $75 \%$ & $25 \%$ & $0 \%$ & 24 & $50 \%$ & $46 \%$ & $4 \%$ & 14 & $71 \%$ & $29 \%$ & $0 \%$ & 33 & $79 \%$ & $21 \%$ & $0 \%$ & 10 & $50 \%$ & $50 \%$ & $0 \%$ \\
\hline & 우 & 3 & $100 \%$ & $0 \%$ & $0 \%$ & 17 & $88 \%$ & $12 \%$ & $0 \%$ & 24 & $42 \%$ & $58 \%$ & $0 \%$ & 14 & $71 \%$ & $29 \%$ & $0 \%$ & 27 & $81 \%$ & $19 \%$ & $0 \%$ & 11 & $45 \%$ & $55 \%$ & $0 \%$ \\
\hline & all & 6 & $100 \%$ & $0 \%$ & $0 \%$ & 37 & $81 \%$ & $19 \%$ & $0 \%$ & 48 & $46 \%$ & $52 \%$ & $2 \%$ & 28 & $71 \%$ & $29 \%$ & $0 \%$ & 60 & $80 \%$ & $20 \%$ & $0 \%$ & 21 & $48 \%$ & $52 \%$ & $0 \%$ \\
\hline \multirow[t]{3}{*}{ VLTPA } & 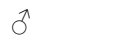 & - & - & - & - & 15 & $100 \%$ & $0 \%$ & $0 \%$ & 11 & $64 \%$ & $36 \%$ & $0 \%$ & 9 & $33 \%$ & $67 \%$ & $0 \%$ & 8 & $88 \%$ & $13 \%$ & $0 \%$ & 13 & $77 \%$ & $23 \%$ & $0 \%$ \\
\hline & q & - & - & - & - & 13 & $92 \%$ & $8 \%$ & $0 \%$ & 12 & $67 \%$ & $33 \%$ & $0 \%$ & 10 & $40 \%$ & $60 \%$ & $0 \%$ & 8 & $88 \%$ & $13 \%$ & $0 \%$ & 11 & $100 \%$ & $0 \%$ & $0 \%$ \\
\hline & all & - & - & - & - & 28 & $96 \%$ & $4 \%$ & $0 \%$ & 23 & $65 \%$ & $35 \%$ & $0 \%$ & 19 & $37 \%$ & $63 \%$ & $0 \%$ & 16 & $88 \%$ & $13 \%$ & $0 \%$ & 24 & $88 \%$ & $13 \%$ & $0 \%$ \\
\hline \multirow[t]{3}{*}{ AT } & $\hat{0}$ & 1 & $100 \%$ & $0 \%$ & $0 \%$ & 7 & $29 \%$ & $14 \%$ & $57 \%$ & 6 & $33 \%$ & $17 \%$ & $50 \%$ & - & - & - & - & 6 & $50 \%$ & $33 \%$ & $17 \%$ & 6 & $17 \%$ & $67 \%$ & $17 \%$ \\
\hline & q & 1 & $100 \%$ & $0 \%$ & $0 \%$ & 5 & $40 \%$ & $20 \%$ & $40 \%$ & 6 & $33 \%$ & $17 \%$ & $50 \%$ & - & - & - & - & 6 & $50 \%$ & $33 \%$ & $17 \%$ & 4 & $25 \%$ & $50 \%$ & $25 \%$ \\
\hline & all & 2 & $100 \%$ & $0 \%$ & $0 \%$ & 12 & $33 \%$ & $17 \%$ & $50 \%$ & 12 & $33 \%$ & $17 \%$ & $50 \%$ & - & - & - & - & 12 & $50 \%$ & $33 \%$ & $17 \%$ & 10 & $20 \%$ & $60 \%$ & $20 \%$ \\
\hline
\end{tabular}

a SEP = socioeconomic position, $\mathrm{PA}=$ physical activity.

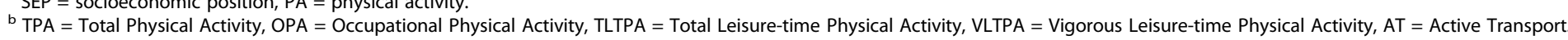

' The symbol ' + ' indicates the positive associations (a high SEP is associated with a high PA), the symbol ' - ' indicates negative associations (a high SEP is associated with a low PA), and ' $O$ ' indicates the neutral

associations (no association found).
$\mathrm{d} a$
$\delta=$ males, $q=$ females, all $=$ genders combined. 


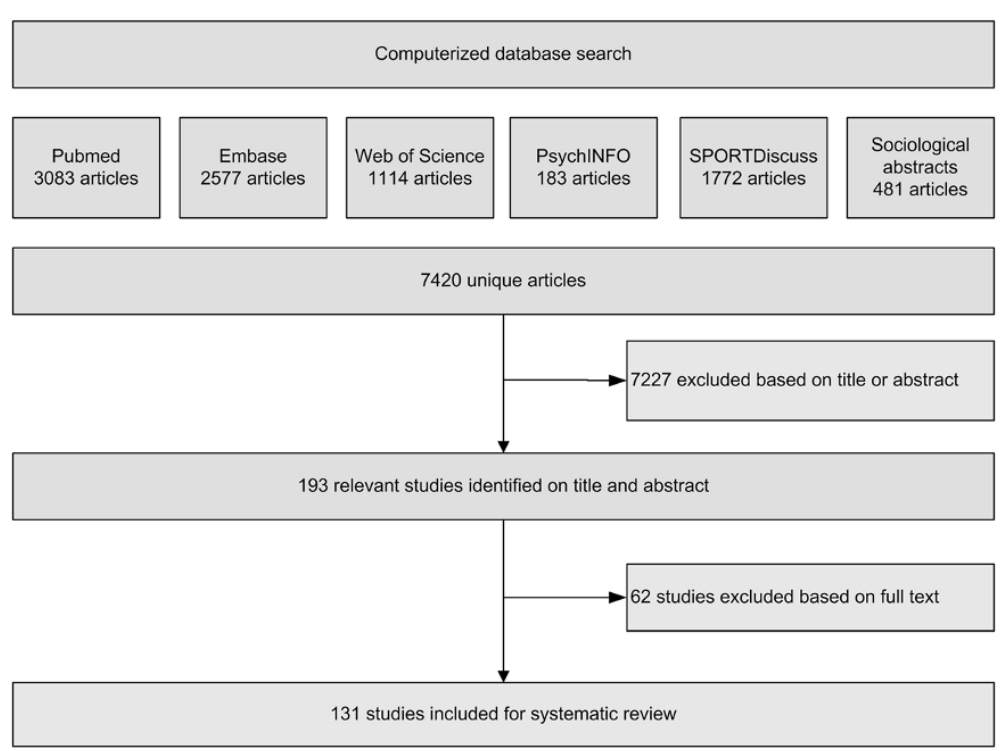

Figure 1 Flowchart of search and selection process.

310 unique associations. The results are presented for total leisure-time PA and vigorous leisure-time PA separately.

\section{Total leisure-time physical activity}

A total of 75 studies reported 200 unique associations (Additional file 1, Table A3) on the association between TLTPA and SEP. Most studies (68\% of associations) showed that people with a higher SEP were more likely to be physically active in their leisure-time, whereas one study reported that a higher SEP was associated with less TLTPA (Table 2). The association between education and TLTPA was reported most frequently and most studies found a positive association (74\%) (Table 2). Men and women differed slightly by the SEP indicator used. For women, the associations between education and TLTPA were mostly positive ( $78 \%$ in women versus $68 \%$ in men), and for men the associations between social class and TLTPA were mostly positive (79\% in men versus $68 \%$ in women). Income showed a more consistent positive association with TLTPA among men (71\% positive) compared to women ( $47 \%$ positive). There were also geographical differences (Table 3). In Scandinavia and the Western European countries, predominantly positive associations were observed $(84 \%$ and $81 \%$ respectively). In Eastern Europe and in the Anglo-Saxon region, only half of the associations were positive ( $46 \%$ and $48 \%$ respectively), with the remaining being null associations.

\section{Vigorous leisure-time physical activity}

The results from the 37 included studies reporting about VLTPA and SEP showed clear socioeconomic inequalities in VLTPA (Additional file 1, Table A4). A total of 84 out of the 110 associations (76\%) were positive, indicating that higher socioeconomic groups were more vigorously physically active during leisure-time than lower socioeconomic groups (Table 2). No studies found a significant inverse association. Income was found to be positively associated with VLTPA more frequently among men (83\%) than among women (67\%) (Table 2). Regarding the other SEP indicators, the results were slightly more pronounced in women. Nearly all studies (96\%) conducted in the Western European region reported that VLTPA was more prevalent among people with a higher SEP (Table 3). In both Scandinavia and in the Anglo-Saxon countries, the positive associations also dominated (both $88 \%$ positive), whereas in Southern Europe about a third of the associations were positive (37\%), the other $63 \%$ being non significant.

\section{Active transport}

There were 11 studies that examined socioeconomic differences in active transport (Additional file 1, Table A5). Two studies distinguished between engaging in active transport (yes/no) and the amount of active transport in a week $[28,29]$. This resulted in a total of 48 associations of which $18(38 \%)$ were positive, $14(29 \%)$ were neutral, and $16(33 \%)$ were negative (Table 2). There were no clear differences by gender, SEP indicator, or geographic region (Tables 2 and 3).

\section{Quality sensitivity analyses}

After excluding all studies that did not report a percentage of response or that did not have a response of at least $50 \%(n=40)$, a total of 91 studies remained in the 
sensitivity analysis. The number of associations decreased from 447 to 313, though patterns remained similar (Additional file 1, Table A6 and Table A7). The main difference was that now all associations between OPA and SEP were negative, compared with $63 \%$ in the main analysis.

Excluding associations that were not at least adjusted for age and gender from the analysis resulted in a total of 342 unique associations (Additional file 1, Table A6 and Table A7). In this restricted set of studies, all associations between OPA and SEP were negative thus accentuating the negative pattern found in the main analysis. All other patterns remained similar.

Finally, excluding the studies with less than 2000 participants $(n=31)$ resulted in an analysis with the remaining 100 studies (Additional file 1, Table A6 and Table A7). The patterns became somewhat more pronounced, since larger studies in general produce more significant associations. In this restricted set of studies, half of the associations for TPA were positive, compared with $40 \%$ in the main analysis. Also the associations in TLTPA and VLTPA were more often positive (77\% and $82 \%$ relatively compared with $68 \%$ and $76 \%$ ). The associations between OPA and SEP were more often negative (77\% compared with 63\%). The pattern for active transport remained similar.

\section{Discussion}

Patterns of socioeconomic inequalities in PA are perhaps more complex than often thought. The direction of socioeconomic inequalities in PA in Europe differs considerably by domain of PA and to some degree by European region and socioeconomic indicator. Since only few studies reported men and women separately, no conclusions about gender differences are warranted.

\section{Domains of physical activity}

Different domains of PA demonstrated different socioeconomic patterns. The most consistent socioeconomic inequalities were found for vigorous leisure-time PA, with the lower SEP groups participating less in vigorous activities like sports than higher SEP groups. For overall leisure-time PA a similar pattern was observed although less articulated. In contrast to PA during leisure time, occupational PA was more frequently reported by lower SEP groups. For total PA and active transport, many studies found a significant association, but they differed considerably in direction.

The absence of a consistent direction in the socioeconomic inequalities in total PA might be caused by the contrasting socioeconomic patterns found for leisuretime PA and occupational PA, that both may make up a large part of total PA. This was nicely illustrated by a study by Lissner and colleagues [30]. They studied leisure-time PA, occupational PA, and PA index (total PA) which was a combined measure of occupational and leisure-time PA. Their results showed that education was positively associated with leisure-time PA and inversely associated with occupational PA. Education and the PA index were not associated since the association between leisure-time PA and occupational PA evened each other out. This mechanism may partly explain the contradictory results with as much negative as positive associations between SEP and total PA, since the association will be determined by the relative influence of leisure-time PA and occupational PA on total PA.

Another question that rises is whether occupational physical activity compensates for not being active during leisure time. A few included studies [31,32] examined socio-economic differences in leisure-time PA while correcting for occupational PA. In the multivariable models, both income and education, and occupational PA were significantly associated with leisure-time PA. These studies indicated that although respondents who were more occupationally active were less active in leisure time, people from lower socio-economic backgrounds were still less physically active compared to high socioeconomic people, even after correcting for occupational PA.

Also, by including occupational PA as an indicator of healthy PA, it is assumed that occupational PA is beneficial to health, however this may not be the case [33]. The few studies that look at associations between occupational PA and mortality or morbidity show no clear pattern. There are studies that report a beneficial effect [34-38], no effect [39], or a detrimental effect [40-43] of occupational PA on cardiovascular diseases and mortality. The health benefits of leisure-time PA and sports are more consistent [34-37,42,44,45]. The different types of activity carried out at work might partly explain these inconsistent findings. For example, Fransson et al. [46] found that walking and standing at work, both aerobic activities, decreases the risk of myocardial infarction, while lifting or carrying at work increases the risk of myocardial infarction. The relation between all aspects of occupational PA and health should be investigated further.

Active transport was studied considerably less often than the other domains of PA and no clear pattern was detected. There were almost equal amounts of studies showing a positive, a null, or a negative association between SEP and active transport. It could be that whether or not one engages in active transport and time spent doing so have different determinants. The two studies that distinguished between participation and time spent in active transport showed for example that participation was not or inversely associated with education while, among the participators, the higher educated spent more 
time in active transport $[28,29]$. The contradictory results may also be explained by factors that influence the association between SEP and active transport. A Dutch and a Belgium study both looked at neighbourhood SEP as an indicator of active transport and found negative associations [23,47]. This could either be an indication that people with a lower SEP are more likely to engage in transport PA or for example, that neighbourhoods with a low SEP are more likely to make people engage in transport PA for example because of a higher density or more connectivity [48]. External factors such as connectivity, density and the availability of public transport might be especially important for active transport PA and more research should be conducted to get a better insight into determinants of active transport.

\section{Types of SEP indicator}

Income, education and occupation reflect different aspects of SEP $[17,18]$. Occupational class appears to be the SEP indicator most sensitive for studying SEP differences in occupational PA. However, the consistent associations found for this indicator may also be due to the definitions used to describe social classes. Because manual jobs are in general considered to be of lower social class, the social class definition is often partly based on having a manual or a non-manual job. This already implies a difference in activities at work.

Inequalities in leisure-time PA and vigorous activity are often thought to be caused by either an educational effect on knowledge about the positive health consequences of $\mathrm{PA}$, or financial possibilities to engage in leisure-time PA, for example to buy PA equipment or to afford memberships or admission rates for sports and PA facilities. The fact that the patterns in inequalities in PA were roughly similar for the different indicators of SEP, including education and income, suggest that it is not one or the other but both may indeed be important. Other factors related to chance and choice of lifestyle [49], such as SEP differences in social or cultural capital [50] or differences in physical environmental opportunities for PA [51,52], may be additional determinants of SEP inequalities in PA. Also, some factors, such as intrapersonal factors, may act as intermediary in the process between SES and PA [52]. In a previous review, Gidlow and colleagues [53] reported that education was stronger associated with PA than income. Although in the present review education was the most frequent studied SEP indicator we could not confirm that the associations of education with PA were also stronger than the associations with the other SEP indicators.

\section{European regions}

A recent study showed that the largest inequalities in obesity prevalence were found in Southern Europe, especially among women, and the smallest in Eastern Europe [7]. In concordance with these findings, we found that the socioeconomic inequalities in PA were less consistent in Eastern Europe for both occupational PA and leisure-time PA. Opposite to what would be expected from the inequalities found in obesity, the inequalities in vigorous leisure-time PA were least pronounced in Southern Europe. This was also found in the few pan-European studies that were included in this review [54-56] and by a recent pan-European study by Mäkinen et al. [57]. A possible explanation could be that general levels of PA are low in these countries [54,57] which would make it harder to detect SEP differences in PA.

\section{Strengths \& limitations}

The main strength of this review is the systematic exploration of different domains of physical activity, different SEP indicators, and geographic regions of Europe. Also, the inclusion of a quality sensitivity analyses strengthens the results. There are, however, also some limitations to be taken into account when interpreting the results.

Like any review of the published literature, the present review may suffer from publication bias [58]. The fact that a substantial numbers of null findings were reported in the reviewed studies may indicate that publication bias may not be severe. Also, some relevant studies may have been missed because only English-language studies that were available in electronic databases and that were published in peer-reviewed journals were included. Moreover, by analyzing the data on the level of the associations instead of the level of studies, more weight was given to studies that reported more than one association. Although this may have influenced conclusions based on all reported associations, this influence was expected to be smaller when subgroups of associations, such as by PA domain and SEP indicator, are considered.

Methodological differences between the included studies, such as the assessment of PA [59], the selection of participants, and the adjustment for confounders, could have influenced the reported associations. Although this probably introduced some noise, the sensitivity analysis showed that the overall patterns seem to be quite stable.

\section{Conclusion}

This review showed that leisure-time PA, and specifically vigorous leisure-time $\mathrm{PA}$, is less prevalent while occupational PA is more prevalent among people with lower SEP. Although there were some regional differences, these inequalities were visible throughout Europe. The contradictory inequalities for total PA may partly be explained by the contrasting socioeconomic patterns found for leisuretime PA and occupational PA. These inconsistent results in total PA indicate that total PA may not be a suitable 
summary measure when investigating inequalities in PA and their effects on morbidity and mortality.

The found inequalities indicate that leisure-time PA should be an important focus in improving physical activity levels and reducing inequalities. However, interventions aimed at improving leisure-time PA in lower socioeconomic groups needs to acknowledge their potential higher levels of occupational PA.

\section{Additional files}

Additional file 1: Table A1. Summary of study findings examining associations between total or usual physical activity (TPA) and SEP. Table A2: Summary of study findings examining associations between occupational physical activity (OPA) and SEP. Table A3: Summary of study findings examining associations between total leisure-time physical activity (TLTPA) and SEP. Table A4: Summary of study findings examining associations between vigorous leisure-time physical activity (VLTPA) and SEP. Table A5: Summary of study findings examining associations between active transport (AT) and SEP. Table A6: Distribution of positive, negative, and null associations by SEP indicator and PA domain in differenet subsets of the reviewed associations. Table A7: Distribution of positive, negative, and null associations by European region and PA domain in differenet subsets of the reviewed associations.

\section{Abbreviations}

PA: Physical Activity; MET: Metabolic Equivalent; SEP: Socioeconomic Position; TPA: Total physical Activity; OPA: Occupational Physical Activity; TLTPA: Total Leisure Time Physical Activity; VLTPA: Vigorous Leisure Time Physical Activity; AT: Active Transport.

\section{Competing interest}

The author(s) declare that they have no competing interests'.

\section{Authors' contributions}

MAB carried out the systematic literature search, the title scanning and selection, the data synthesis, and the drafting of the manuscript. CBMK assisted in the title selection and the data synthesis. She also helped to interpret the results and critically reviewed and improved the manuscript. $K G, F J V L$, and AEK designed the study, helped to interpret the results, and critically reviewed and improved the manuscript. JB and $A B$ helped to interpret the results and critically reviewed and improved the manuscript. All authors read and approved the final manuscript.

\section{Acknowledgements}

This work was supported by the Commission of the European Communities [SP5A-CT-2006-044128 "Health Promotion through Obesity Prevention across Europe (HOPE): an integrated analysis to support European health policy"]. The study does not necessarily reflect the Commission's views and in no way anticipates the Commission's future policy in this area.

\section{Author details}

${ }^{1}$ Department of Public Health, Erasmus MC, Rotterdam, Netherlands. ${ }^{2}$ School of Public Health/Institute for Health and Biomedical Innovation, Queensland University of Technology, Brisbane, Australia. ${ }^{3}$ School of Medicine, University of Sydney, Sydney, Australia. ${ }^{4}$ Department of Epidemiology \& Biostatistics and the EMGO Institute for Health and Care Research, VU University Medical Centre, Amsterdam, Netherlands. ${ }^{5}$ Department of Public Health, AMC, University of Amsterdam, Amsterdam, Netherlands.

Received: 28 February 2012 Accepted: 4 September 2012 Published: 19 September 2012

\section{References}

1. Mackenbach JP, Stirbu I, Roskam AJ, Schaap MM, Menvielle G, Leinsalu M, Kunst AE: Socioeconomic inequalities in health in 22 European countries. N Engl J Med 2008, 358:2468-2481.
2. Stirbu I, Kunst AE, Bopp M, Leinsalu M, Regidor E, Esnaola S, Costa G, Martikainen P, Borrell C, Deboosere P, et al: Educational inequalities in avoidable mortality in Europe. J Epidemiol Community Health 2010, 64:913-920

3. Stringhini S, Sabia S, Shipley M, Brunner E, Nabi H, Kivimaki M, SinghManoux A: Association of socioeconomic position with health behaviors and mortality. JAMA 2010, 303:1159-1166.

4. Menvielle $G$, Boshuizen $H$, Kunst AE, Dalton SO, Vineis P, Bergmann MM, Hermann S, Ferrari P, Raaschou-Nielsen O, Tjønneland A, et al: The role of smoking and diet in explaining educational inequalities in lung cancer incidence. J Natl Cancer Inst 2009, 101:321-330.

5. Huisman M, Kunst AE, Mackenbach JP: Educational inequalities in smoking among men and women aged 16 years and older in 11 European countries. Tob Control 2005, 14:106-113.

6. McLaren L: Socioeconomic status and obesity. Epidemiol Rev 2007, 29:29-48.

7. Roskam AJ, Kunst AE, Van Oyen H, Demarest S, Klumbiene J, Regidor E, Helmert U, Jusot F, Dzurova D, Mackenbach JP: Comparative appraisal of educational inequalities in overweight and obesity among adults in 19 European countries. Int J Epidemiol 2010, 39:392-404.

8. Seidell JC: Epidemiology of obesity. Semin Vasc Med 2005, 5:3-14

9. Molarius A, Seidell JC, Sans S, Tuomilehto J, Kuulasmaa K: Educational level, relative body weight, and changes in their association over 10 years: an international perspective from the WHO MONICA Project. Am J Public Health 2000, 90:1260-1268.

10. Branca F, Nikogosian H, Lobstein T: Challenge of Obesity in the WHO European Region and the Strategies for Response. Denmark: World Health Organization; 2007.

11. Giskes $K$, Avendaňo M, Brug J, Kunst AE: A systematic review of studies on socioeconomic inequalities in dietary intakes associated with weight gain and overweight/obesity conducted among European adults. Obes Rev 2010, 11:413-429.

12. Centers for Disease Control and Prevention: Physical activity and health: a report of the Surgeon General. Washington DC: U.S. Department of Health and Human services; 1996.

13. Warburton DE, Nicol CW, Bredin SS: Health benefits of physical activity: the evidence. Can Med Assoc J 2006, 174:801-809.

14. Pate RR, Pratt M, Blair SN, Haskell WL, Macera CA, Bouchard C, Buchner D, Ettinger W, Heath GW, King AC, et al: Physical activity and public health. A recommendation from the Centers for Disease Control and Prevention and the American College of Sports Medicine. JAMA 1995, 273:402-407.

15. Allender S, Foster C, Boxer A: Occupational and nonoccupational physical activity and the social determinants of physical activity: results from the Health Survey for England. J Phys Act Health 2008, 5:104-116.

16. Popham F, Mitchell R: Relation of employment status to socioeconomic position and physical activity types. Prev Med 2007, 45:182-188.

17. Geyer S, Hemström Ö, Peter R, Vågerö D: Education, income, and occupational class cannot be used interchangeably in social epidemiology. Empirical evidence against a common practice. J Epidemiol Community Health 2006, 60:804-810.

18. Galobardes B, Lynch J, Smith GD: Measuring socioeconomic position in health research. Br Med Bull 2007, 81-82:21-37.

19. Rose D: Official Social Classifications in the UK. Soc Res Updat 1995, 9:1.

20. Eikemo TA, Huisman M, Bambra C, Kunst AE: Health inequalities according to educational level in different welfare regimes: a comparison of 23 European countries. Sociol Health IIIn 2008, 30:565-582.

21. Ferrera M: The 'Southern Model' of Welfare in Social Europe. Journal of European Social Policy 1996, 6:17-37.

22. Ball K, Crawford D: Socioeconomic status and weight change in adults: a review. Soc Sci Med 2005, 60:1987-2010.

23. Van Dyck D, Cardon G, Deforche B, Sallis JF, Owen N, De Bourdeaudhuij I: Neighborhood SES and walkability are related to physical activity behavior in Belgian adults. Prev Med 2010, 50:S74-S79.

24. Craig $C L$, Marshall AL, Sjöström M, Bauman AE, Booth ML, Ainsworth BE, Pratt M, Ekelund U, Yngve A, Sallis JF, Oja P: International physical activity questionnaire: 12-country reliability and validity. Med Sci Sports Exerc 2003, 35:1381-1395.

25. Taylor HL, Jacobs DR Jr, Schucker B, Knudsen J, Leon AS, Debacker G: A questionnaire for the assessment of leisure time physical activities. J Chronic Dis 1978, 31:741-755. 
26. Pereira MA, FitzerGerald SJ, Gregg EW, Joswiak ML, Ryan WJ, Suminski RR, Utter AC, Zmuda JM: A collection of Physical Activity Questionnaires for health-related research. Med Sci Sports Exerc 1997, 29:S1-S205.

27. Wendel-Vos GCW, Schuit AJ, Saris WHM, Kromhout D: Reproducibility and relative validity of the short questionnaire to assess health-enhancing physical activity. J Clin Epidemiol 2003, 56:1163-1169.

28. Kwaśniewska M, Kaczmarczyk-Chałas K, Pikala M, Broda, Kozakiewicz K, Pajak A, Tykarski A, Zdrojewski T, Drygas W: Socio-demographic and lifestyle correlates of commuting activity in Poland. Prev Med 2010, 50:257-261.

29. Adams J: Prevalence and socio-demographic correlates of "active transport" in the UK: analysis of the UK time use survey 2005. Prev Med 2010, 50:199-203.

30. Lissner L, Bengtsson C, Björkelund C, Wedel H: Physical activity levels and changes in relation to longevity. A prospective study of Swedish women. Am J Epidemiol 1996, 143:54-62.

31. Drygas W, Kwaśniewska M, Kaleta D, Pikala M, Bielecki W, Głuszek J, Zdrojewski T, Pajak A, Kozakiewicz K, Broda G: Epidemiology of physical inactivity in Poland: prevalence and determinants in a former communist country in socioeconomic transition. Public Health 2009 123:592-597.

32. Kaleta $D$, Jegier $A$ : Occupational energy expenditure and leisure-time physical activity. Int J Occup Med Environ Health 2005, 18:351-356.

33. Holtermann A, Hansen JV, Burr H, Søgaard K, Sjøgaard G: The health paradox of occupational and leisure-time physical activity. Br J Sports Med 2012, 69:485-492.

34. Wang $Y$, Tuomilehto J, Jousilahti $P$, Antikainen $R$, Mähönen M, Katzmarzyk PT, Hu G: Occupational, commuting, and leisure-time physical activity in relation to heart failure among finnish men and women. J Am Coll Cardiol 2010, 56:1140-1148

35. Salonen JT, Puska P, Tuomilehto J: Physical activity and risk of myocardia infarction, cerebral stroke and death: a longitudinal study in Eastern Finland. Am J Epidemiol 1982, 115:526-537.

36. Hu G, Jousilahti P, Borodulin K, Barengo NC, Lakka TA, Nissinen A, Tuomilehto J: Occupational, commuting and leisure-time physical activity in relation to coronary heart disease among middle-aged Finnish men and women. Atherosclerosis 2007, 194:490-497.

37. Hu G, Qiao Q, Silventoinen K, Eriksson JG, Jousilahti P, Lindström J, Valle TT, Nissinen A, Tuomilehto J: Occupational, commuting, and leisure-time physical activity in relation to risk for Type 2 diabetes in middle-aged Finnish men and women. Diabetologia 2003, 46:322-329.

38. Steeves JA, Bassett DR Jr, Thompson DL, Fitzhugh EC: Relationships of occupational and non-occupational physical activity to abdominal obesity. Int J Obes 2012, 36:100-106.

39. Sisson SB, Camhi SM, Church TS, Martin CK, Tudor-Locke C, Bouchard C, Earnest CP, Smith SR, Newton RL Jr, Rankinen T, Katzmarzyk PT: Leisure time sedentary behavior, occupational/domestic physical activity, and metabolic syndrome in U.S. men and women. Metab Syndr Relat Disord 2009, 7:529-536

40. Stender M, Hense HW, Döring A, Keil U: Physical activity at work and cardiovascular disease risk: results from the MONICA Augsburg study. Int J Epidemiol 1993, 22:644-650.

41. Kristal-Boneh E, Harari G, Melamed S, Froom P: Association of physical activity at work with mortality in Israeli industrial employees: the CORDIS study. J Occup Environ Med 2000, 42:127-135.

42. Suadicani $P$, Hein $H O$, Gyntelberg F: Socioeconomic status and ischaemic heart disease mortality in middle-aged men: importance of the duration of follow-up. The Copenhagen Male Study. Int J Epidemio/ 2001, 30:248-255.

43. Krause N, Brand RJ, Kaplan GA, Kauhanen J, Malla S, Tuomainen TP, Salonen JT: Occupational physical activity, energy expenditure and 11-year progression of carotid atherosclerosis. Scand J Work Environ Health 2007, 33:405-424

44. Behre CJ, Bergström G, Schmidt CB: Increasing leisure time physical activity is associated with less prevalence of the metabolic syndrome in healthy middle-aged men. Angiology 2011, 62:509-512.

45. Savela S, Koistinen P, Tilvis RS, Strandberg AY, Pitkälä KH, Salomaa W, Miettinen TA, Strandberg TE: Leisure-time physical activity, cardiovascular risk factors and mortality during a 34-year follow-up in men. Eur $\mathrm{J}$ Epidemiol 2010, 25:619-625.

46. Fransson E, De Faire U, Ahlbom A, Reuterwall C, Hallqvist J, Alfredsson L: The risk of acute myocardial infarction: interactions of types of physical activity. Epidemiology 2004, 15:573-582.
47. van Lenthe FJ, Brug J, Mackenbach JP: Neighbourhood inequalities in physical inactivity: the role of neighbourhood attractiveness, proximity to local facilities and safety in the Netherlands. Soc Sci Med 2005, 60:763-775

48. Rundle A, Roux AV, Free LM, Miller D, Neckerman KM, Weiss CC: The urban built environment and obesity in New York City: a multilevel analysis. Am J Health Promot 2007, 21:326-334.

49. Cockerham WC, Rütten A, Abel T: Conceptualizing contemporary health lifestyles. Sociological Quarterly 1997, 38:321-342.

50. Abel T: Cultural capital and social inequality in health. J Epidemiol Community Health 2008, 62:e13.

51. Kamphuis CB, van Lenthe FJ, Giskes K, Huisman M, Brug J, Mackenbach JP. Socioeconomic differences in lack of recreational walking among older adults: the role of neighbourhood and individual factors. Int J Behav Nutr Phys Act 2009, 6:1

52. Kamphuis CB, Van Lenthe FJ, Giskes K, Huisman M, Brug J, Mackenbach JP: Socioeconomic status, environmental and individual factors, and sports participation. Med Sci Sports Exerc 2008, 40:71-81.

53. Gidlow C, Johnston LH, Crone D, Ellis N, James D: A systematic review of the relationship between socio-economic position and physical activity Health Educ J 2006, 65:338-367.

54. Martinez-Gonzalez MA, Varo JJ, Santos JL, De Irala J, Gibney M, Kearney J, Martinez JA: Prevalence of physical activity during leisure time in the European Union. Med Sci Sports Exerc 2001, 33:1142-1146.

55. Van Tuyckom C, Scheerder J: Sport for All? Insight into stratification and compensation mechanisms of sporting activity in the 27 European Union member states. Sport, Education and Society 2010, 15:495-512.

56. Varo JJ, Martínez-González MA, De Irala-Estévez J, Kearney J, Gibney M, Martínez JA: Distribution and determinants of sedentary lifestyles in the European Union. Int J Epidemiol 2003, 32:138-146.

57. Mäkinen TE, Sippola R, Borodulin K, Rahkonen O, Kunst A, Klumbiene J, Regidor E, Ekholm O, Mackenbach J, Prättälä R: Explaining educational differences in leisure-time physical activity in Europe: the contribution of work-related factors. Scand J Med Sci Sports 2012, 22:439-447.

58. Dickersin K, Min Y-I, Meinert CL: Factors Influencing Publication of Research Results: Follow-up of Applications Submitted to Two Institutional Review Boards. JAMA 1992, 267:374-378.

59. van Poppel MN, Chinapaw MJ, Mokkink LB, van Mechelen W, Terwee CB: Physical activity questionnaires for adults: a systematic review of measurement properties. Sports Med 2010, 40:565-600.

60. Ståhl T, Rütten A, Nutbeam D, Bauman A, Kannas L, Abel T, Lüschen G, Rodriquez DJ, Vinck J, van der Zee J: The importance of the social environment for physically active lifestyle-results from an international study. Soc Sci Med 2001, 52:1-10

61. Van Tuyckom C, Scheerder J: Sport for all? Social stratification of recreational sport activities in the EU-27. Kinesiologia Slovenica 2008, 14:54-63.

62. Van Tuyckom C, Scheerder J: A multilevel analysis of social stratification patterns of leisure-time physical activity among Europeans. Science \& Sports 2010, 25:304-311.

63. Addor V, Wietlisbach V, Narring F, Michaud PA: Cardiovascular risk factor profiles and their social gradient from adolescence to age 74 in a Swiss region. Prev Med 2003, 36:217-228.

64. Bertrais S, Preziosi P, Mennen L, Galan P, Hercberg S, Oppert JM: Sociodemographic and geographic correlates of meeting current recommendations for physical activity in middle-aged French adults: the Supplementation en Vitamines et Mineraux Antioxydants (SUVIMAX) Study. Am J Public Health 2004, 94:1560-1566.

65. Chaix B, Chauvin P: Tobacco and alcohol consumption, sedentary lifestyle and overweightness in France: A multilevel analysis of individual and area-level determinants. Eur J Epidemiol 2003, 18:531-538.

66. Dragano N, Bobak M, Wege N, Peasey A, Verde PE, Kubinova R, Weyers S, Moebus S, Möhlenkamp S, Stang A, et al: Neighbourhood socioeconomic status and cardiovascular risk factors: a multilevel analysis of nine cities in the Czech Republic and Germany. BMC Public Health 2007, 7:255.

67. Drieskens S, Van Oyen H, Demarest S, Van der Heyden J, Gisle L, Tafforeau J: Multiple risk behaviour: increasing socio-economic gap over time? Eur J Public Health 2010, 20:634-639.

68. Galobardes B, Costanza MC, Bernstein MS, Delhumeau C, Morabia A: Trends in risk factors for lifestyle-related diseases by socioeconomic position in Geneva, Switzerland, 1993-2000: health inequalities persist. Am J Public Health 2003, 93:1302-1309. 
69. Meyer K, Rezny L, Breuer C, Lamprecht M, Stamm HP: Physical activity of adults aged 50 years and older in Switzerland. Soz Praventivmed 2005, 50:218-229

70. Nocon M, Keil T, Willich SN: Education, income, occupational status and health risk behaviour. J Public Health 2008, 15:401-405.

71. van Oort FV, van Lenthe FJ, Mackenbach JP: Cooccurrence of lifestyle risk factors and the explanation of education inequalities in mortality: results from the GLOBE study. Prev Med 2004, 39:1126-1134.

72. Rathmann W, Haastert B, Icks A, Giani G, Holle R, Meisinger C, Mielck A, Group KS: Sex differences in the associations of socioeconomic status with undiagnosed diabetes mellitus and impaired glucose tolerance in the elderly population: the KORA Survey 2000. Eur J Public Health 2005, 15:627-633.

73. Ribet $C$, Lang $T$, Zins $M$, Bingham A, Ferrières J, Arveiler D, Amouyel $P$, Bonenfant S, Chastang JF, Goldberg M, Ducimetière P: Do cardiovascular risk factors in men depend on their spouses' occupational category? Eur J Epidemiol 2001, 17:347-356.

74. Scheerder J, Vanreusel B, Taks M, Renson R: Social Sports Stratification in Flanders 1969-1999: Intergenerational Reproduction of Social Inequalities? International Review for the Sociology of Sport 2002, 37:219-245.

75. Scheerder J, Vanreusel B, Taks M: Stratification patterns of active sport involvement among adults: Social change and persistence. International Review for the Sociology of Sport 2005, 40:139-162.

76. Schneider S, Becker S: Prevalence of physical activity among the working population and correlation with work-related factors: results from the first German National Health Survey. J Occup Health 2005, 47:414-423.

77. Verdaet D, Dendale P, De Bacquer D, Delanghe J, Block P, De Backer G: Association between leisure time physical activity and markers of chronic inflammation related to coronary heart disease. Atherosclerosis 2004, 176:303-310.

78. De Vries H, Vant Riet J, Spigt M, Metsemakers J, Van den Akker M, Vermunt JK, Kremers S: Clusters of lifestyle behaviors: results from the Dutch SMILE study. Prev Med 2008, 46:203-208.

79. Wagner A, Simon C, Evans A, Ducimetière $P$, Bongard V, Montaye M, Arveiler D, Group PS: Physical activity patterns in 50-59 year men in France and Northern Ireland. Associations with socio-economic status and health behaviour. Eur J Epidemiol 2003, 18:321-329.

80. Frömel K, Mitáš J, Kerr J: The associations between active lifestyle, the size of a community and SES of the adult population in the Czech Republic. Health Place 2009, 15:447-454.

81. Jurakić $D$, Pedišić $Z$, Andrijašević M: Physical activity of Croatian population: cross-sectional study using International Physical Activity Questionnaire. Croat Med J 2009, 50:165-173.

82. Kaleta $D$, Jegier $A$ : Predictors of inactivity in the working-age population Int J Occup Med Environ Health 2007, 20:175-182.

83. Leskošek B, Doupona M, Moćnik R, Jošt B: Sport participation in the Republic of Slovenia and its relation to selected socio-demographic variables./Beteiligung an Sport in der Republik Slowenien und deren Zusammenhang mit den Ausgewaehlten sozio-demographischen Variablen./Relacije sudjelovanja u sportskim aktivnostima u Repbulici Slovenijis izabranim socio-demografskim varijablama. Kinesiology 2002, 34:41-49.

84. Mišigoj-Durakoviæ M, Heimer S, Matkoviæ BR, Ružiæ L, Prskalo I: Physical activity of urban adult population: questionnaire study. Croat Med J 2000, 41:428-432.

85. Nowak MA: Relationships between the perception of the influence of physical activity on health and women's engagement in exercise or their physical passivity. Polish Journal of Sport \& Tourism 2010, 17:179-184.

86. Paulik E, Bóka F, Kertész A, Balogh S, Nagymajtényi L: Determinants of health-promoting lifestyle behaviour in the rural areas of Hungary. Health Promot Int 2010, 25:277-288.

87. Pomerleau J, McKee M, Robertson A, Vaasc S, Kadziauskiene K, Abaravicius A, Bartkeviciute R, Pudule I, Grinberga D: Physical inactivity in the Baltic countries. Prev Med 2000, 31:665-672

88. Puska P, Helasoja V, Prättälä R, Kasmel A, Klumbiene J: Health behaviour in Estonia, Finland and Lithuania 1994-1998. Standardized comparison. Eur J Public Health 2003, 13:11-17.

89. Shapo L, Pomerleau J, McKee M: Physical inactivity in a country in transition: a population-based survey in Tirana City, Albania. Scand J Public Health 2004, 32:60-67.
90. Stelmach W, Kaczmarczyk-Chałas K, Bielecki W, Drygas W: The impact of income, education and health on lifestyle in a large urban population of Poland (Cindi programme). Int J Occup Med Environ Health 2004, 17:393-401.

91. Zaletel-Kragelj L, Fras Z, Zakotnik JM: Results of CINDI health monitor surveys in Slovenia as a tool for development of effective healthy nutrition and physical activity intervention programmes. $J$ Public Health 2007, 14:110-118.

92. Artazcoz L, Borrell C, Benach J, Cortès I, Rohlfs I: Women, family demands and health: the importance of employment status and socio-economic position. Soc Sci Med 2004, 59:263-274.

93. Bolívar J, Daponte A, Rodríguez M, Sánchez JJ: The Influence of Individual, Social and Physical Environment Factors on Physical Activity in the Adult Population in Andalusia, Spain. Int J Environ Res Public Health 2010, 7:60-77.

94. Borrell C, Domínguez-Berjón F, Pasarín MI, Ferrando J, Rohlfs I, Nebot M: Social inequalities in health related behaviours in Barcelona. J Epidemiol Community Health 2000, 54:24-30.

95. Borrell C, Rue M, Pasarín MI, Rohlfs I, Ferrando J, Fernandez E: Trends in social class inequalities in health status, health-related behaviors, and health services utilization in a Southern European urban area (1983-1994). Prev Med 2000, 31:691-701.

96. De Vogli R, Gnesotto R, Goldstein M, Andersen R, Cornia GA: The lack of social gradient of health behaviors and psychosocial factors in Northern Italy. Soz Praventivmed 2005, 50:197-205.

97. Gal DL, Santos AC, Barros H: Leisure-time versus full-day energy expenditure: a cross-sectional study of sedentarism in a Portuguese urban population. BMC Public Health 2005, 5:16

98. Lera-López F, Rapún-Gárate M: Sports Participation versus Consumer expenditure on Sport: Different Determinants and Strategies in Sports Management. European Sport Management Quarterly 2005, 5:167-186.

99. Meseguer CM, Galán I, Herruzo R, Zorrilla B, Rodríguez-Artalejo F: Leisuretime physical activity in a southern European mediterranean country: adherence to recommendations and determining factors. Rev Esp Cardiol 2009, 62:1125-1133.

100. Panagiotakos DB, Pitsavos C, Chrysohoou C, Vlismas K, Skoumas Y, Palliou K, Stefanadis C: Dietary habits mediate the relationship between socioeconomic status and CVD factors among healthy adults: the ATTICA study. Public Health Nutr 2008, 11:1342-1349.

101. Panagiotakos DB, Pitsavos C, Chrysohoou C, Vlismas K, Skoumas Y, Palliou K, Stefanadis $C$ : The effect of clinical characteristics and dietary habits on the relationship between education status and 5-year incidence of cardiovascular disease: the ATTICA study. Eur J Nutr 2008, 47:258-265.

102. Pascual C, Regidor E, Astasio P, Ortega P, Navarro P, Domínguez V: The association of current and sustained area-based adverse socioeconomic environment with physical inactivity. Soc Sci Med 2007, 65:454-466.

103. Pascual C, Regidor E, Martínez D, Elisa Calle M, Domínguez V: Socioeconomic environment, availability of sports facilities, and jogging, swimming and gym use. Health Place 2009, 15:553-561.

104. Pitsavos C, Panagiotakos DB, Lentzas Y, Stefanadis C: Epidemiology of leisure-time physical activity in socio-demographic, lifestyle and psychological characteristics of men and women in Greece: the ATTICA study. BMC Public Health 2005, 5:37.

105. Santos R, Santos MP, Ribeiro JC, Mota J: Physical Activity and Other Lifestyle Behaviors in a Portuguese Sample of Adults: Results From the Azorean Physical Activity and Health Study. J Phys Act Health 2009, 6:750-759.

106. Schröder H, Rohlfs I, Schmelz EM, Marrugat J, for the REGIDOR investigators: Relationship of socioeconomic status with cardiovascular risk factors and lifestyle in a Mediterranean population. Eur J Nutr 2004, 43:77-85.

107. Ali SM, Lindström M: Psychosocial work conditions, unemployment, and leisure-time physical activity: A population-based study. Scand J Public Health 2006, 34:209-216.

108. Andersen LB, Schnohr P, Schroll M, Hein HO: All-cause mortality associated with physical activity during leisure time, work, sports, and cycling to work. Arch Intern Med 2000, 160:1621-1628.

109. Barengo NC, Nissinen A, Pekkarinen $\mathrm{H}$, Halonen P, Tuomilehto J: Twentyfive-year trends in lifestyle and socioeconomic characteristics in Eastern Finland. Scand J Public Health 2006, 34:437-444.

110. Bergman P, Grjibovski AM, Hagströmer M, Bauman A, Sjöstrom M: Adherence to physical activity recommendations and the influence of 
socio-demographic correlates - a population-based cross-sectional study. BMC Public Health 2008, 8:367.

111. Borodulin K, Laatikainen T, Lahti-Koski M, Jousilahti P, Lakka TA: Association of age and education with different types of leisure-time physical activity among 4437 Finnish adults. J Phys Act Health 2008, 5:242-251.

112. Cubbin C, Sundquist K, Ahlén H, Johansson SE, Winkleby MA, Sundquist J: Neighborhood deprivation and cardiovascular disease risk factors: protective and harmful effects. Scand J Public Health 2006, 34:228-237.

113. Engström L-M: Who is physically active? Cultural capital and sports participation from adolescence to middle ageâ€"a 38-year follow-up study. Physical Education \& Sport Pedagogy 2008, 13:319-343.

114. Häkkinen $U$, Järvelin MR, Rosenqvist $G$, Laitinen J: Health, schooling and lifestyle among young adults in Finland. Health Econ 2006, 15:1201-1216.

115. Henriksson KM, Lindblad U, Ågren B, Nilsson-Ehle P, Råstam L: Associations between unemployment and cardiovascular risk factors varies with the unemployment rate: the Cardiovascular Risk Factor Study in Southern Sweden (CRISS). Scand J Public Health 2003, 31:305-311.

116. Kivimäki M, Lawlor DA, Davey Smith G, Kouvonen A, Virtanen M, Elovainio $M$, Vahtera J: Socioeconomic position, co-occurrence of behavior-related risk factors, and coronary heart disease: the Finnish Public Sector Study. Am J Public Health 2007, 97:874-879.

117. Korniloff K, Häkkinen A, Kautiainen $H$, Koponen $H$, Peltonen M, Mäntyselkä $P$, Oksa H, Kampman O, Vanhala M: Leisure-time physical activity and metabolic syndrome plus depressive symptoms in the FIN-D2D survey. Prev Med 2010, 51:466-470.

118. Laaksonen M, Lahelma E, Prättälä R: Associations among health-related behaviours: sociodemographic variation in Finland. Soz Praventivmed 2002, 47:225-232

119. Laaksonen M, Talala K, Martelin T, Rahkonen O, Roos E, Helakorpi S, Laatikainen T, Prättälä R: Health behaviours as explanations for educational level differences in cardiovascular and all-cause mortality: a follow-up of 60000 men and women over 23 years. Eur J Public Health 2008, 18:38-43.

120. Lagerros YT, Bellocco R, Adami HO, Nyrén O: Measures of physical activity and their correlates: the Swedish National March Cohort. Eur J Epidemiol 2009, 24:161-169.

121. Leijon ME, Stark-Ekman D, Nilsen P, Ekberg K, Walter L, Ståhle A, Bendtsen P: Is there a demand for physical activity interventions provided by the health care sector? Findings from a population survey. BMC Public Health 2010, 10:34

122. Lindström M, Hanson BS, Östergren PO: Socioeconomic differences in leisure-time physical activity: the role of social participation and social capital in shaping health related behaviour. Soc Sci Med 2001, 52:441-451.

123. Lindström M, Isacsson SO, Merlo J: Increasing prevalence of overweight, obesity and physical inactivity: two population-based studies 1986 and 1994. Eur J Public Health 2003, 13:306-312.

124. Lindström M, Moghaddassi M, Merlo J: Social capital and leisure time physical activity: a population based multilevel analysis in Malmo, Sweden. J Epidemiol Community Health 2003, 57:23-28.

125. Mäkinen T, Borodulin K, Laatikainen T, Fogelholm M, Prättälä R: Twenty-five year socioeconomic trends in leisure-time and commuting physical activity among employed Finns. Scand J Med Sci Sports 2009, 19:188-197.

126. Mäkinen T, Kestilä L, Borodulin K, Martelin T, Rahkonen O, Leino-Arjas P, Prättälä R: Occupational class differences in leisure-time physical inactivity - contribution of past and current physical workload and other working conditions. Scand J Work Environ Health 2010, 36:62-70.

127. Mäkinen T, Kestilä L, Borodulin K, Martelin T, Rahkonen O, Prättälä R: Effects of childhood socio-economic conditions on educational differences in leisure-time physical activity. Eur J Public Health 2010, 20:346-353.

128. Mäkinen TE, Borodulin K, Tammelin TH, Rahkonen $\mathrm{O}$, Laatikainen T, Prättälä $R$ : The effects of adolescence sports and exercise on adulthood leisuretime physical activity in educational groups. Int J Behav Nutr Phys Act 2010, 7:27

129. Molarius A: The contribution of lifestyle factors to socioeconomic differences in obesity in men and women - a population-based study in Sweden. Eur J Epidemiol 2003, 18:227-234.

130. Nielsen TL, Wraae K, Brixen K, Hermann AP, Andersen M, Hagen C: Prevalence of overweight, obesity and physical inactivity in 20- to 29year-old, Danish men. Relation to sociodemography, physical dysfunction and low socioeconomic status: the Odense Androgen Study. Int J Obes 2006, 30:805-815.
131. Norman A, Bellocco R, Vaida F, Wolk A: Total physical activity in relation to age, body mass, health and other factors in a cohort of Swedish men. Int J Obes Relat Metab Disord 2002, 26:670-675.

132. Novak M, Ahlgren C, Hammarström A: A life-course approach in explaining social inequity in obesity among young adult men and women. Int J Obes 2006, 30:191-200.

133. Orsini N, Bellocco R, Bottai M, Pagano M, Wolk A: Correlates of total physical activity among middle-aged and elderly women. Int J Behav Nutr Phys Act 2007, 4:16.

134. Osler M, Gerdes LU, Davidsen M, Brønnum-Hansen H, Madsen M, Jørgensen T, Schroll M: Socioeconomic status and trends in risk factors for cardiovascular diseases in the Danish MONICA population, 1982-1992. J Epidemiol Community Health 2000, 54:108-113.

135. Osler M, Clausen JO, Ibsen KK, Jensen GB: Social influences and low leisure-time physical activity in young Danish adults. Eur J Public Health 2001, 11:130-134.

136. Osler M, Godtfredsen NS, Prescott E: Childhood social circumstances and health behaviour in midlife: the Metropolit 1953 Danish male birth cohort. Int J Epidemiol 2008, 37:1367-1374.

137. Petersen CB, Thygesen LC, Helge JW, Grønbæk M, Tolstrup JS: Time trends in physical activity in leisure time in the Danish population from 1987 to 2005. Scand J Public Health 2010, 38:121-128.

138. Piro FN, Næss O, Claussen B: Area deprivation and its association with health in a cross-sectional study: are the results biased by recent migration? Int J Equity Health 2007, 6:10.

139. Pudaric S, Sundquist J, Johansson SE: Major risk factors for cardiovascular disease in elderly migrants in Sweden. Ethn Health 2000, 5:137-150.

140. Pulkki L, Kivimäki M, Elovainio M, Viikari J, Keltikangas-Järvinen L: Contribution of socioeconomic status to the association between hostility and cardiovascular risk behaviors: a prospective cohort study. Am J Epidemiol 2003, 158:736-742.

141. Pulkki L, Kivimäki M, Keltikangas-Järvinen L, Elovainio M, Leino M, Viikari J: Contribution of adolescent and early adult personality to the inverse association between education and cardiovascular risk behaviours: prospective population-based cohort study. Int J Epidemiol 2003, 32:968-975.

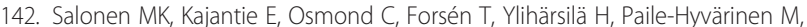
Barker DJ, Eriksson JG: Prenatal and childhood growth and leisure time physical activity in adult life. Eur J Public Health 2010, 21:719-724.

143. Schnohr C, Højbjerre L, Riegels M, Ledet L, Larsen T, Schultz-Larsen K, Petersen L, Prescott E, Grønæk M: Does educational level influence the effects of smoking, alcohol, physical activity, and obesity on mortality? A prospective population study. Scand J Public Health 2004, 32:250-256.

144. Simonen RL, Videman T, Kaprio J, Levälahti E, Battié MC: Factors associated with exercise lifestyle - A study of monozygotic twins. Int J Sports Med 2003, 24:499-505.

145. Sjögren K, Stjernberg L: A gender perspective on factors that influence outdoor recreational physical activity among the elderly. BMC Geriatr 2010, 10:34

146. Strand BH, Tverdal A: Can cardiovascular risk factors and lifestyle explain the educational inequalities in mortality from ischaemic heart disease and from other heart diseases? 26 year follow up of 50,000 Norwegian men and women. J Epidemiol Community Health 2004, 58:705-709.

147. Strandhagen E, Berg C, Lissner L, Nunez L, Rosengren A, Torén K, Thelle DS: Selection bias in a population survey with registry linkage: potential effect on socioeconomic gradient in cardiovascular risk. Eur J Epidemiol 2010, 25:163-172

148. Suadicani P, OleHein H, Gyntelberg F: Lifestyle, social class, and obesity-the Copenhagen Male Study. Eur J Cardiovasc Prev Rehabil 2005, 12:236-242.

149. Tammelin T, Näyhä S, Laitinen J, Rintamäki H, Järvelin MR: Physical activity and social status in adolescence as predictors of physical inactivity in adulthood. Prev Med 2003, 37:375-381.

150. Wemme KM, Rosvall M: Work related and non-work related stress in relation to low leisure time physical activity in a Swedish population. J Epidemiol Community Health 2005, 59:377-379.

151. Adams J: The mediating role of time perspective in socio-economic inequalities in smoking and physical activity in older English adults J Health Psychol 2009, 14:794-799.

152. Amuzu A, Carson C, Watt HC, Lawlor DA, Ebrahim S: Influence of area and individual lifecourse deprivation on health behaviours: findings from the British Women's Heart and Health Study. Eur J Cardiovasc Prev Rehabil 2009, 16:169-173. 
153. Bartley M, Fitzpatrick R, Firth D, Marmot M: Social distribution of cardiovascular disease risk factors: change among men in England 1984-1993. J Epidemiol Community Health 2000, 54:806-814.

154. Bartley M, Martikainen P, Shipley M, Marmot M: Gender differences in the relationship of partner's social class to behavioural risk factors and social support in the Whitehall II Study. Soc Sci Med 2004, 59:1925-1936.

155. Chaudhury M, Shelton N: Physical activity among 60-69-year-olds in England: knowledge, perception, behaviour and risk factors. Ageing \& Society 2010, 30:1343-1355.

156. Ecob R, Macintyre S: Small area variations in health related behaviours; do these depend on the behaviour itself, its measurement, or on personal characteristics? Health Place 2000, 6:261-274

157. Harrison RA, McElduff $P$, Edwards R: Planning to win: Health and lifestyles associated with physical activity amongst 15,423 adults. Public Health 2006, 120:206-212

158. Heslop P, Smith GD, Macleod J, Hart C: The socioeconomic position of employed women, risk factors and mortality. Soc Sci Med 2001, 53:477-485.

159. Hillsdon M, Lawlor DA, Ebrahim S, Morris JN: Physical activity in older women: associations with area deprivation and with socioeconomic position over the life course: observations in the British Women's Heart and Health Study. J Epidemiol Community Health 2008, 62:344-350.

160. Lahelma E, Lallukka T, Laaksonen M, Martikainen P, Rahkonen O, Chandola T, Head J, Marmot M, Kagamimori S, Tatsuse T, Sekine M: Social class differences in health behaviours among employees from Britain, Finland and Japan: The influence of psychosocial factors. Health Place 2010, 16:61-70.

161. Livingstone MB, Robson PJ, McCarthy S, Kiely M, Harrington K, Browne P, Galvin M, Wareham NJ, Rennie KL: Physical activity patterns in a nationally representative sample of adults in Ireland. Public Health Nutr 2001, 4:1107-1116.

162. Lunn PD: The sports and exercise life-course: a survival analysis of recall data from Ireland. Soc Sci Med 2010, 70:711-719.

163. Mein GK, Shipley MJ, Hillsdon M, Ellison GT, Marmot MG: Work, retirement and physical activity: cross-sectional analyses from the Whitehall II Study. Eur J Public Health 2005, 15:317-322.

164. Mullineaux DR, Barnes CA, Barnes EF: Factors affecting the likelihood to engage in adequate physical activity to promote health. J Sports Sci 2001, 19:279-288.

165. Mutrie N, Hannah MK: Some work hard while others play hard: The achievement of current recommendations for physical activity levels at work, at home, and in leisure time in the West of Scotland. Int I Health Promot Edu 2004, 42:109-117.

166. Myint PK, Luben RN, Welch AA, Bingham SA, Wareham NJ, Khaw KT: Effect of age on the relationship of occupational social class with prevalence of modifiable cardiovascular risk factors and cardiovascular diseases. A population-based cross-sectional study from European Prospective Investigation into Cancer - Norfolk (EPIC-Norfolk). Gerontology 2006, 52:51-58.

167. Poortinga W: Associations of physical activity with smoking and alcohol consumption: a sport or occupation effect? Prev Med 2007, 45:66-70.

168. Popham F, Mitchell R: Leisure time exercise and personal circumstances in the working age population: longitudinal analysis of the British household panel survey. J Epidemiol Community Health 2006, 60:270-274.

169. Popham F: Intergenerational social class stability and mobility are associated with large absolute differences in adult participation in sport and exercise. Br J Sports Med 2010, 44:1184-1185.

170. Stamatakis E, Chaudhury M: Temporal trends in adults' sports participation patterns in England between 1997 and 2006: the Health Survey for England. Br J Sports Med 2008, 42:601-608.

171. Wardle J, Griffith J: Socioeconomic status and weight control practices in British adults. J Epidemiol Community Health 2001, 55:185-190.

172. Wardle J, Steptoe A: Socioeconomic differences in attitudes and beliefs about healthy lifestyles. J Epidemiol Community Health 2003, 57:440-443.

173. Watt HC, Carson C, Lawlor DA, Patel R, Ebrahim S: Influence of life course socioeconomic position on older women's health behaviors: findings from the British Women's Heart and Health Study. Am J Public Health 2009, 99:320-327.

\section{doi:10.1186/1479-5868-9-116}

Cite this article as: Beenackers et al.: Socioeconomic inequalities in occupational, leisure-time, and transport related physical activity among European adults: A systematic review. International Journal of Behavioral

Nutrition and Physical Activity 2012 9:116.

\section{Submit your next manuscript to BioMed Central and take full advantage of:}

- Convenient online submission

- Thorough peer review

- No space constraints or color figure charges

- Immediate publication on acceptance

- Inclusion in PubMed, CAS, Scopus and Google Scholar

- Research which is freely available for redistribution 